\title{
SIMULTANEOUS HIGHER-ORDER OPTICAL FLOW ESTIMATION AND DECOMPOSITION*
}

\author{
JING YUAN ${ }^{\dagger}$, CHRISTOPH SCHNÖRR $^{\dagger}$, AND GABRIELE STEIDL ${ }^{\ddagger}$
}

\begin{abstract}
We study the estimation and decomposition of optical flows from highly nonrigid motions. To this end, recent methods from image decomposition into structural and textural parts are combined with variational optical flow estimation. The approaches we suggest amount to minimizing discrete convex functionals using second-order cone programming. Higher-order regularization is necessary in order to accurately recover important flow structure like vortices, and to incorporate key physical properties such as vanishing divergence. For proper discretization, we apply the finite mimetic difference method, which preserves the identities fulfilled by the continuous differential operators. Numerical examples demonstrate the feasibility of the complex approaches.
\end{abstract}

Key words. optical flow estimation, optical flow decomposition, divergence-free flows, higherorder regularization, mimetic finite difference method, convex optimization, dual optimization techniques, second-order cone programming

AMS subject classifications. 68Y10, 65K10, 90C25, 76-04

DOI. $10.1137 / 060660709$

1. Introduction. The representation, estimation, and analysis of nonrigid motion is relevant to many scenarios in computer vision, medical imaging, remote sensing, and experimental fluid dynamics. Especially in fluid dynamics, sophisticated measurement techniques including pulsed laser light sheets and modern CCD cameras, as well as dedicated hardware, enable the recording of high-resolution image sequences that reveal the evolution of spatio-temporal structures of unsteady flows [25]. In this connection, an important research problem is to develop variational approaches that render flow estimation from image sequences into a well-posed and numerically stable problem while preserving small-scale flow structures that are important for empirical investigations of turbulent phenomena.

To this end, we investigate a novel class of variational flow estimation schemes by combining higher-order flow regularization with recent techniques developed for nonsmooth image decomposition-see below for a more detailed exposition. As a result, we obtain variational approaches that not only allow for estimating fluid flow from image sequences but simultaneously yield a decomposition of the flow into coherent spatio-temporal flow patterns and small-scale structures.

In the following, we briefly describe the respective basic ideas in a continuous setting. In the remainder of the paper, we will derive and investigate discrete approaches using the so-called mimetic finite difference method developed by Hyman and Shashkov [17], which preserves the integral identities fulfilled by the corresponding continuous integral operators.

Image decomposition. In image denoising one is typically interested in removing noise without destroying important structures such as edges. This goal cannot be

\footnotetext{
* Received by the editors May 24, 2006; accepted for publication (in revised form) March 14, 2007; published electronically October 5, 2007.

http://www.siam.org/journals/sisc/29-6/66070.html

${ }^{\dagger}$ CVGPR-Group, University of Mannheim, 68131 Mannheim, Germany (\{yuanjing,schnoerr\}@ uni-mannheim.de).

${ }^{\ddagger}$ Institute of Mathematics, University of Mannheim, 68131 Mannheim, Germany (steidl@math. uni-mannheim.de).
} 
achieved with linear filters, e.g., by minimizing

$$
J(f):=\|g-f\|_{L_{2}(\Omega)}^{2}+\lambda \int_{\Omega}|\nabla f|^{2} \mathrm{~d} x \mathrm{~d} y
$$

for a given noisy image $g(x, y)$ in $\Omega \subset \mathbb{R}^{2}$. The regularizer incorporates the quadratic function $\Phi(s)=s^{2}$ with $s=|\nabla f|$. Via the Euler-Lagrange equation this variational approach can be related to a linear diffusion equation. As a consequence, the solution $f$ smooths $g$ in a completely homogeneous way and therefore blurs semantically important signal structures. To overcome this drawback a variety of nonlinear methods have been proposed. One of the frequently applied approaches replaces the function in the regularization term by $\Phi(s)=s$ and thus penalizes larger deviations of $|\nabla f|$ not as hard as the quadratic function:

$$
J(f):=\frac{1}{2}\|g-f\|_{L_{2}(\Omega)}^{2}+\lambda \int_{\Omega}|\nabla f| \mathrm{d} x \mathrm{~d} y .
$$

This functional was first considered by Rudin, Osher, and Fatemi [26]. For $f$ having partial derivatives in $L_{1}$ the regularizer coincides with the TV seminorm of $f$,

$$
\|f\|_{\mathrm{TV}}:=\int|\nabla f| \mathrm{d} x \mathrm{~d} y .
$$

In contrast to the linear approach (1.1) we will refer to this method as the $T V$ approach. Based on the dual TV norm, the so-called G norm, this denoising model was enlarged for the decomposition of given images $g$ into a structural (cartoon) part $f^{s}$ and a textural part $f^{t}$ as

$$
\begin{aligned}
J\left(f^{s}, f^{t}\right) & =\left\|g-\left(f^{s}+f^{t}\right)\right\|_{L_{2}(\Omega)}^{2}+\lambda \int_{\Omega}\left|\nabla f^{s}\right| \mathrm{d} x \mathrm{~d} y, \\
\text { s.t. } & \left\|f^{t}\right\|_{\mathrm{G}} \leq \delta .
\end{aligned}
$$

For a more sophisticated treatment of the TV and G norms, we refer to $[11,21]$. Meanwhile there exist various numerical realizations of (1.3), e.g., [3, 24, 32, 34]. Although not considered in this paper, we note that there exist other models including other than Gaussian white noise by using the $L_{1}$ norm in the data fitting term [5, 23], noise as a third decomposition component by applying Besov norms [4], or frame and TV/frame approaches [10, 28] from harmonic analysis. Moreover, second-order derivatives were incorporated into the regularizer to avoid, for example, staircasing effects; see, e.g., [8, 29].

In this paper, we are interested in the decomposition of vector fields rather than scalar images. Specifically, we want to deal with optical flow fields arising, e.g., in experimental fluid dynamics.

Optical flow estimation. Instead of a single image let us consider an image sequence $\{g(x, y, t): t \in[0, T]\}$ with a time parameter $t$. A common assumption is that image intensities are preserved over time:

$$
g\left(x\left(t_{1}\right), y\left(t_{1}\right), t_{1}\right)=g\left(x\left(t_{2}\right), y\left(t_{2}\right), t_{2}\right), \quad t_{2}>t_{1} .
$$

Generalizations to other constraints exist but are not relevant for our present investigation. The linearized version of the gray value constancy assumption (1.4) yields the optical flow constraint

$$
g_{x} u_{1}+g_{y} u_{2}+g_{t}=\nabla g \cdot u+g_{t}=0
$$


where the optical flow field $u=\left(u_{1}, u_{2}\right)^{\top}:=(\dot{x}, \dot{y})^{\top}$ denotes the instantaneous velocities of image elements.

Obviously, (1.5) cannot be solved pointwise because at each location and time point it consists of solving a single scalar equation for two scalar unknowns. To overcome this so-called aperture problem additional requirements have to be imposed. Instead of (1.5) we consider the least squares approximation

$$
F(u):=\left\|\nabla g \cdot u+g_{t}\right\|_{L_{2}(\Omega)}^{2} .
$$

In 1981, Horn and Schunck [16] pioneered the field of regularization methods for optical flow computation by suggesting minimization of the functional

$$
J(u)=F(u)+\lambda \int_{\Omega}\left|\nabla u_{1}\right|^{2}+\left|\nabla u_{2}\right|^{2} \mathrm{~d} x \mathrm{~d} y .
$$

We will refer to this widely used approach as the Horn-Schunck model. Obviously, the Horn-Schunck model suffers from the same drawbacks as the linear image filtering model (1.1). The solution creates blurry optic flow fields where the blur appears also across important flow discontinuities. One way to overcome this limitation consists again if using another function in the regularizer, e.g.,

$$
J(u)=\frac{1}{2} F(u)+\lambda \int_{\Omega}\left(\left|\nabla u_{1}\right|^{2}+\left|\nabla u_{2}\right|^{2}\right)^{\frac{1}{2}} \mathrm{~d} x \mathrm{~d} y,
$$

as counterpart to the TV model (1.2); cf. [2, 13, 15].

In this paper we focus on nonrigid motion analysis. In particular, we are interested in the representation of motion by components that capture different physical aspects, e.g., solenoidal (divergence-free) flows. Referring again to experimental fluid dynamics, for example, the extraction of coherent flow structures which are immersed into additional motion components at different spatial scales [19] poses a challenge for image sequence analysis. However, this goal cannot be achieved using first-order derivatives in the regularizer. Rather, based on early work on second-order regularizers constraining the gradients of the flows' divergence and curl [1, 9, 14, 30], instead of (1.7) we deal with the functional

$$
J(u)=F(u)+\lambda_{d} \int_{\Omega}|\nabla \operatorname{div} u|^{2} \mathrm{~d} x \mathrm{~d} y+\lambda_{c} \int_{\Omega}|\nabla \operatorname{curl} u|^{2} \mathrm{~d} x \mathrm{~d} y+\gamma \int_{\partial \Omega}\left(\partial_{\mathrm{n}} u\right)^{2} \mathrm{~d} s
$$

where the boundary is incorporated for stability reasons; cf. [35, 37]. A key property of this approach is that due to the second order of the flow derivatives involved, the regularizing terms do not penalize the magnitude of the basic flow components involving first-order derivatives, i.e., divergence and curl. To further motivate this approach consider Figure 1.1, which shows the estimation of a solenoidal flow field by first- and second-order approaches. The figure clearly demonstrates the superiority of the div-curl model.

Finally, related to the TV model (1.8), we deal with

$$
J(u)=\frac{1}{2} F(u)+\lambda_{d} \int_{\Omega}|\nabla \operatorname{div} u| \mathrm{d} x \mathrm{~d} y+\lambda_{c} \int_{\Omega}|\nabla \operatorname{curl} u| \mathrm{d} x \mathrm{~d} y+\frac{\gamma}{2} \int_{\partial \Omega}\left(\partial_{\mathrm{n}} u\right)^{2} \mathrm{~d} s,
$$

involving regularizing terms that are of second order, as in (9), and that are additionally suited to preserve jumps of the divergence and the curl of a flow field, 


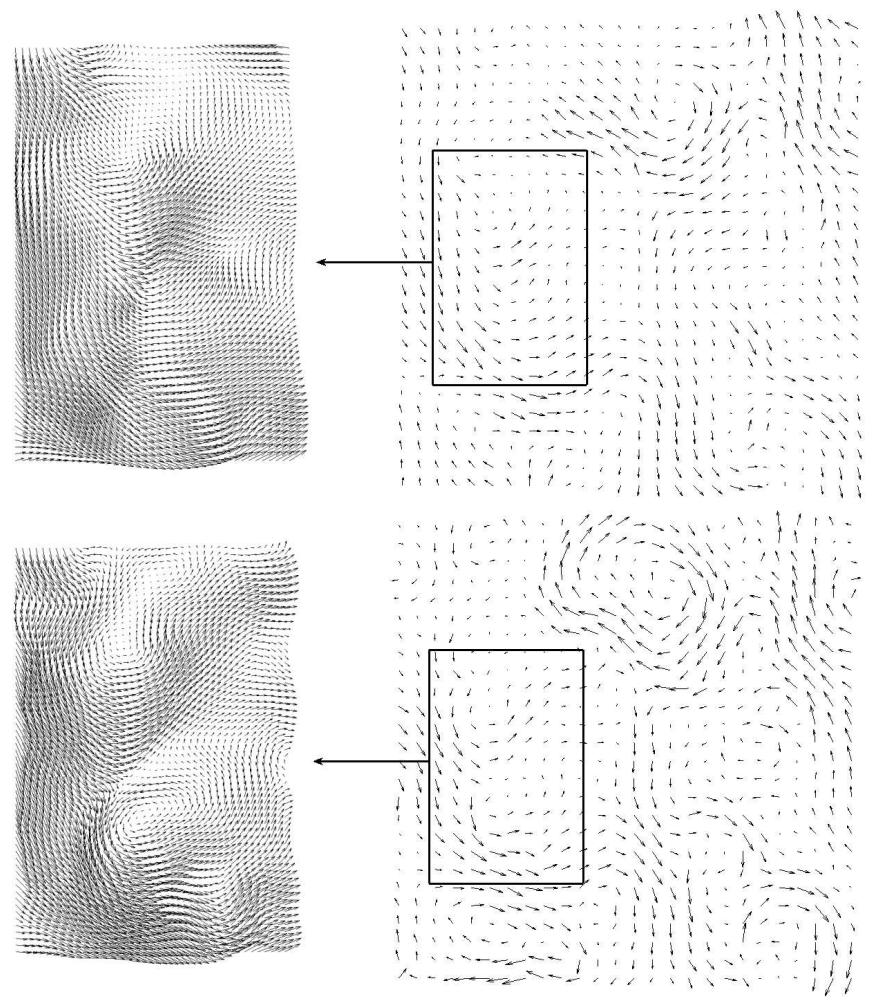

FIG. 1.1. Estimation of a typical solenoidal flow field u. Top: Restored flow based on the Horn-Schunck model (1.7). Bottom: Restored flow based on the second-order model (1.9). Vortex structures are better recovered by the div-curl approach (bottom); cf. [35].

respectively, by utilizing the TV-norm. As in image decomposition we will extend this estimation approach to the decomposition of motion vector fields into physically relevant components at different scales by using a discrete equivalent of the $\mathrm{G}$ norm. Moreover, we will study the feasibility of an extension to the simultaneous estimation and decomposition of optical flows.

Organization of the paper. We start by introducing our discrete setting based on the mimetic finite difference method in section 2 . We define scalar and vector fields on primal and dual grids together with appropriate norms. Then we introduce the firstorder operators, where we focus on their matrix representation using tensor product notation. We show how integral identities such as the Gaussian integral identity and the Helmholtz decomposition carry over to the discrete situation. Finally, we define discrete TV and G norms with respect to the primal and dual grid. Based on these definitions and results we consider the flow estimation task in section 3. Here we are interested in the linear versus the TV approach. While the linear method was already treated in [35], the higher-order TV approach is novel. In section 4, we deal with flow decomposition by the TV-G norm model. To this end we have to introduce a decomposition of the optical flow into components with constant divergence and curl and variable components which can be further decomposed into a "structural" part and a "textural" part. In fact, these parts comprise flow patterns at different scales. In section 5, we combine the flow estimation and the decomposition model in 
order to solve both tasks simultaneously. In sections 3,4 , and 5 we especially focus on the practically relevant case of solenoidal flows. Some parts of sections 4 and 5 were previously announced at a conference [36]. Section 6 provides the numerical algorithms to solve the convex optimization problems posed in sections 3,4 , and 5 . We use second-order cone programming (SOCP) $[12,20]$. Finally, we demonstrate the feasibility of our approaches by numerical examples in section 7 .

2. Discretization. For our discrete versions of the functionals we need a careful discretization which preserves the identities satisfied by the continuous operators as, e.g., div $=-\nabla^{*}$, curl $\nabla=0$, the Gaussian integral identity, and the Helmholtz decomposition. For discretizing the relevant spaces and differential operators we apply the mimetic finite difference method introduced by Hyman and Shashkov in [17]. This method defines the corresponding discrete operators of $\nabla$, div, and curl simultaneously on two grids. These grids, which we call the primal and dual grid, are shifted with respect to each other by half a pixel.

Primal and dual scalar/vector fields. Corresponding to the grids, we define the following scalar fields and vector fields:

$H_{P}$ : space of scalar fields on vertices,

$H_{S}$ : space of vector fields defined normal to sides,

$H_{V}$ : space of scalar field on cells,

$H_{E}$ : space of vector fields defined tangential to sides.

By $H_{P}^{o}, H_{S}^{o}$, and $H_{E}^{o}$ we denote the corresponding spaces with zero boundary elements. Finally, let $H_{V+\partial V}$ and $H_{E+\partial E}$ be the extended version of $H_{V}$ and $H_{E}$, respectively, to the boundary. If our primal grid consists of $m \times n$ vertices, then $\operatorname{dim} H_{P}=m n$, $\operatorname{dim} H_{V}=(m-1)(n-1), \operatorname{dim} H_{S}=m(n-1)+n(m-1)$, and $\operatorname{dim} H_{E}=(m-1)(n-$ $2)+(n-1)(m-2)$. The fields are illustrated in Figure 2.1.
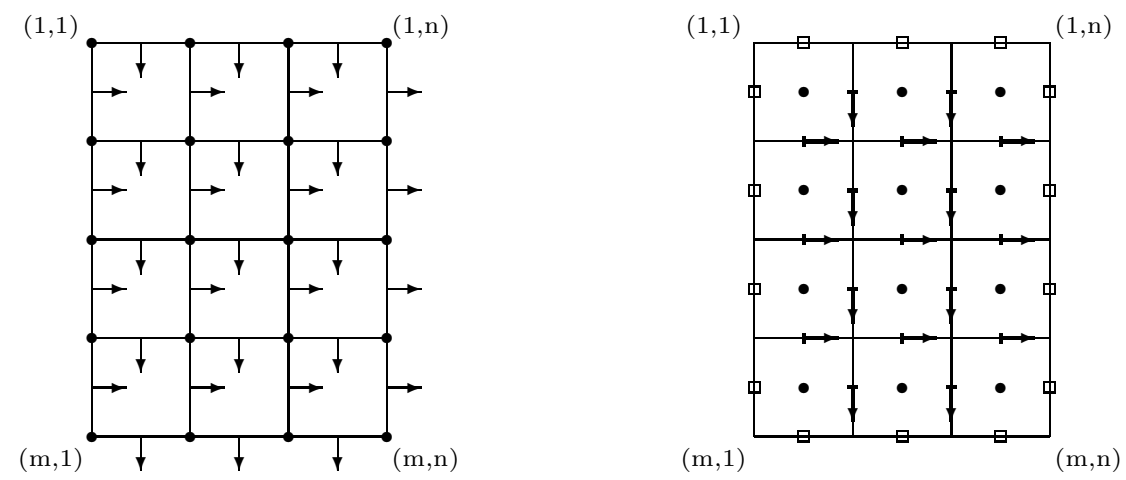

FIG. 2.1. Primal and dual grid. Left: Spaces $H_{P}$ (dots) and $H_{S}$ (arrows). Right: Spaces $H_{V}$ (dots), $H_{\partial V}$ (squares), and $H_{E}$ (arrows) for $m=5$ and $n=4$.

While $H_{P}$ and $H_{V}$ are equipped with the usual $\ell_{2}$ inner product, the inner products on $H_{S}$ and $H_{E}$ are defined with respect to the cells. To this end, we consider the elements of $H_{S}$ associated with the elements of $H_{V}$ by

$$
u_{V_{i+\frac{1}{2}, j+\frac{1}{2}}}:=\frac{1}{\sqrt{2}}\left(u_{i, j+\frac{1}{2}}, u_{i+1, j+\frac{1}{2}}, u_{i+\frac{1}{2}, j}, u_{i+\frac{1}{2}, j+1}\right)^{\mathrm{T}}
$$

for $i=1, \ldots, m-1, j=1, \ldots, n-1$. Then we define a dotproduct $\cdot: H_{S} \times H_{S} \rightarrow H_{V}$ 
by

$$
u \cdot v:=\left((u \cdot v)_{i+\frac{1}{2}, j+\frac{1}{2}}\right)_{i, j=1}^{m-1, n-1}=\left(\left\langle u_{V_{i+\frac{1}{2}, j+\frac{1}{2}}}, v_{V_{i+\frac{1}{2}, j+\frac{1}{2}}}\right\rangle\right)_{i, j=1}^{m-1, n-1},
$$

where $\langle\cdot, \cdot\rangle$ denotes the usual inner product on $\ell_{2}$. Now the inner product on the vector field $H_{S}$ is given by

$$
\langle u, v\rangle_{H_{S}}:=\sum_{i=1}^{m-1} \sum_{j=1}^{n-1}(u \cdot v)_{i+\frac{1}{2}, j+\frac{1}{2}}
$$

and the corresponding norm by

$$
\begin{aligned}
\|u\|_{H_{S}}^{2} & :=\left\|\left(\left\|u_{V_{i+\frac{1}{2}, j+\frac{1}{2}}}\right\|_{2}\right)_{i, j=1}^{m-1, n-1}\right\|_{2}^{2} \\
& =\frac{1}{2} \sum_{i=1}^{m-1} \sum_{j=1}^{n-1}\left(u_{i, j+\frac{1}{2}}^{2}+u_{i+1, j+\frac{1}{2}}^{2}+u_{i+\frac{1}{2}, j}^{2}+u_{i+\frac{1}{2}, j+1}^{2}\right) .
\end{aligned}
$$

Related to the $\ell_{1}$ norm we define

$$
\begin{aligned}
|u|_{H_{S}} & :=\left\|\left(\left\|u_{V_{i+\frac{1}{2}, j+\frac{1}{2}}}\right\|_{2}\right)_{i, j=1}^{m-1, n-1}\right\|_{1} \\
& =\frac{1}{\sqrt{2}} \sum_{i=1}^{m-1} \sum_{j=1}^{n-1} \sqrt{u_{i, j+\frac{1}{2}}^{2}+u_{i+1, j+\frac{1}{2}}^{2}+u_{i+\frac{1}{2}, j}^{2}+u_{i+\frac{1}{2}, j+1}^{2}} .
\end{aligned}
$$

Similarly, we associate elements of $u \in H_{E}$ with elements of $H_{P}^{o}$ by

$$
u_{P_{i, j}}:=\frac{1}{\sqrt{2}}\left(u_{i, j-\frac{1}{2}}, u_{i, j+\frac{1}{2}}, u_{i-\frac{1}{2}, j}, u_{i+\frac{1}{2}, j}\right)^{\mathrm{T}}
$$

for $i=2, \ldots, m-1, j=2, \ldots, n-1$, and set

$$
\|u\|_{H_{E}}^{2}:=\left\|\left(\left\|u_{P_{i, j}}\right\|_{2}\right)_{i, j=2}^{m-1, n-1}\right\|_{2}^{2}, \quad|u|_{H_{E}}:=\left\|\left(\left\|u_{P_{i, j}}\right\|_{2}\right)_{i, j=2}^{m-1, n-1}\right\|_{1} .
$$

Primal and dual operators. We use the following discrete versions of the first-order operators $\nabla$, div, and curl with respect to the primal and dual grid:

$$
\mathbb{G r a d}: H_{P} \rightarrow H_{E+\partial E}, \quad \mathbb{G r a d}{ }^{\perp}: H_{P} \rightarrow H_{S}
$$

and

$$
\begin{array}{lll}
\mathbb{G}: H_{P}^{o} \rightarrow H_{E}^{o}, & \mathbb{D} i v: H_{S} \rightarrow H_{V}, & \mathbb{G}^{\perp}: H_{P}^{o} \rightarrow H_{S}^{o}, \\
\mathbb{G}: H_{V} \rightarrow H_{S}^{o}, & \mathbb{D} i v: H_{E}^{o} \rightarrow H_{P}^{o}, & \mathbb{C} u r l: H_{S}^{o} \rightarrow H_{P}^{o} .
\end{array}
$$

Reshaping the scalar/vector fields columnwise into vectors of appropriate lengths, these operators act on the corresponding vector spaces as matrices which can be described by using the first-order forward difference matrix

$$
D_{m}:=\left(\begin{array}{rrrrrrr}
-1 & 1 & 0 & \ldots & 0 & 0 & 0 \\
0 & -1 & 1 & \ldots & 0 & 0 & 0 \\
& & \ddots & \ddots & \ddots & & \\
0 & 0 & 0 & \ldots & -1 & 1 & 0 \\
0 & 0 & 0 & \ldots & 0 & -1 & 1
\end{array}\right) \in \mathbb{R}^{m-1, m}
$$


and the tensor product $\otimes$ of matrices. Then we have

$$
\mathbb{G r a d}=\left(\begin{array}{c}
I_{n} \otimes D_{m} \\
D_{n} \otimes I_{m}
\end{array}\right), \quad \mathbb{G} r a d^{\perp}=\left(\begin{array}{c}
-D_{n} \otimes I_{m} \\
I_{n} \otimes D_{m}
\end{array}\right),
$$

which explains the notation ${ }^{\perp}$. The operator $\mathbb{G}^{\perp}$ is just the restriction of $\mathbb{G r a d} d^{\perp}$ to $H_{P}^{o}$. Although $\mathbb{G}$ is not the restriction of $\mathbb{G r} r d$ to $H_{P}^{o}$, we will again use the notation $\perp$. More precisely, we define

$$
\begin{gathered}
\mathbb{G}=\left(\begin{array}{c}
I_{n-2} \otimes D_{m-2} \\
D_{n-2} \otimes I_{m-2}
\end{array}\right), \quad \mathbb{G}^{\perp}=\left(\begin{array}{c}
D_{n-1}^{\mathrm{T}} \otimes I_{m-2} \\
-I_{n-2} \otimes D_{m-1}^{\mathrm{T}}
\end{array}\right), \\
\mathbb{D} i v=\left(I_{n-1} \otimes D_{m}, D_{n} \otimes I_{m-1}\right),
\end{gathered}
$$

where $I_{n}$ denotes the $n \times n$ identity matrix and the matrices are considered without the zero rows/columns due to embedding. Then we have on the dual grid

$$
\overline{\mathbb{G}}=-\left(\left.\mathbb{D} i\right|_{\left.\right|_{H_{S}^{o}}}\right)^{*}, \quad \overline{\mathbb{D} i v}=-\mathbb{G}^{*}, \quad \overline{\mathbb{C} u r l}=\left(\mathbb{G}^{\perp}\right)^{*},
$$

where the adjoint operator corresponds to the transposed matrices. Using properties of the tensor product of matrices, we obtain that $\operatorname{curl} \nabla=0$ reads as

$$
\overline{\mathbb{C} u r l} \overline{\mathbb{G}}=0, \quad \mathbb{D} i v \mathbb{G}^{\perp}=-\overline{\mathbb{G}}^{*} \overline{\mathbb{C} u r l}^{*}=0 .
$$

We extend the operator $\overline{\mathbb{G}}$ to $H_{V+\partial V}$ by incorporating the boundary elements $H_{\partial V}$ into the forward differences. Since the distance of a boundary point to its neighboring inner point is only $\frac{1}{2}$, we have to multiply the difference filter $(-1,1)$ at the boundary by 2 . The matrix of the resulting operator,

$$
\overline{\mathbb{G}}_{1}: H_{V+\partial V} \rightarrow H_{S},
$$

is given in the appendix. Finally, we define another extension of $\overline{\mathbb{G}}$ in one of the following ways:

- apply a linear extrapolation operator from $H_{V}$ to $H_{V+\partial V}$ and then $\overline{\mathbb{G}}_{1}$,

- apply $\overline{\mathbb{G}}$ and then a constant extrapolation from $H_{S}^{o}$ to $H_{S}$.

Both procedures result in the same operator,

$$
\overline{\mathbb{G}}_{2}: H_{V} \rightarrow H_{S},
$$

given in matrix form in the appendix.

Discrete integral identities. Next we are interested in a discrete version of the Gaussian integral identity $\int_{\Omega} \operatorname{div} u \mathrm{~d} x=\int_{\partial \Omega} n \cdot u \mathrm{~d} s$. To this end, we introduce the boundary operator

$$
\mathbb{B}:=\left(\begin{array}{cc}
I_{n-1} \otimes B_{m} & 0 \\
0 & B_{n} \otimes I_{m-1}
\end{array}\right),
$$

where 0 are zero matrices of appropriate sizes and

$$
B_{m}:=\left(\begin{array}{rrrrr}
-1 & 0 & \ldots & 0 & 0 \\
0 & 0 & \ldots & 0 & 1
\end{array}\right) \in \mathbb{R}^{2, m} .
$$


Then the mimetic Gaussian integral identity becomes

$$
1_{\operatorname{dim} H_{V}}^{\mathrm{T}} \mathbb{D} i v u=1_{\operatorname{dim} \partial H_{V}}^{\mathrm{T}} \mathbb{B} u \text {. }
$$

We will apply the following discrete version of the Helmholtz decomposition.

Proposition 2.1 (mimetic Helmholtz decomposition). Any $u \in H_{S}$ can be decomposed into an irrotational part $u_{\mathrm{irr}}$ and a soleniodal part $u_{\mathrm{sol}}$ by

$$
u=u_{\mathrm{irr}}+u_{\mathrm{sol}}=\overline{\mathbb{G}}_{1} \psi+\mathbb{G}^{\perp} \varphi,
$$

where $\varphi \in H_{P}^{o}$ is uniquely determined and $\psi \in H_{V+\partial V}$ is uniquely determined up to an additive constant. Moreover, the decomposition is orthogonal in the sense that $\left\langle\overline{\mathbb{G}}_{1} \psi, \mathbb{G}^{\perp} \varphi\right\rangle_{H_{S}}=0$.

Proof. By $(2.2)$ and since $\overline{\mathbb{G}}_{1}$ considered as mapping onto $H_{S}^{o}$ equals $\overline{\mathbb{G}}$, we see that

$$
\overline{\mathbb{C} u r l} u_{\text {irr }}=\overline{\mathbb{C} u r l} \overline{\mathbb{G}}_{1} \psi=0, \quad \mathbb{D} i v u_{\text {sol }}=\mathbb{D} i v \mathbb{G}^{\perp} \varphi=0 .
$$

Therefore we have indeed an irrotational and a solenoidal part.

By definition of the matrices it is easy to check that $\operatorname{dim} \mathcal{R}\left(\overline{\mathbb{G}}_{1}\right)=\operatorname{dim} H_{V+\partial V}-1=$ $m n+m+n-4$, and $\operatorname{dim} \mathcal{R}\left(\mathbb{G}^{\perp}\right)=\operatorname{dim} H_{P}^{o}=(m-2)(n-2)$ so that $\operatorname{dim} \mathcal{R}\left(\overline{\mathbb{G}}_{1}\right)+$ $\operatorname{dim} \mathcal{R}\left(\mathbb{G}^{\perp}\right)=\operatorname{dim} H_{S}$. Here $\mathcal{R}$ denotes the range of the operator. By $(2.1)$ and $(2.2)$ we obtain that

$$
\left\langle\overline{\mathbb{G}}_{1} \psi, \mathbb{G}^{\perp} \varphi\right\rangle_{H_{S}}=\left\langle\overline{\mathbb{C} u r l} \overline{\mathbb{G}}_{1} \psi, \varphi\right\rangle_{H_{P}^{o}}=0,
$$

and consequently $H_{S}=\mathcal{R}\left(\overline{\mathbb{G}}_{1}\right) \oplus \mathcal{R}\left(\mathbb{G}^{\perp}\right)$ with the orthogonal sum $\oplus$.

Since $\mathbb{G}^{\perp}$ has full rank, the potential $\varphi$ is uniquely determined by $u_{\text {sol }}$. Since the kernel of $\overline{\mathbb{G}}_{1}$ is given by $\mathcal{N}\left(\overline{\mathbb{G}}_{1}\right)=\left\{c 1_{\operatorname{dim} H_{V+\partial V}}: c \in \mathbb{R}\right\}$ we have that $\psi$ is uniquely determined by $u_{\text {irr }}$ up to an element from the kernel. This completes the proof.

Conversely, assume for $u \in H_{S}$ that

$$
\begin{aligned}
\rho & =\mathbb{D} i v u=\triangle_{d} \psi, \\
\omega & =\overline{\mathbb{C} u r l} u=\triangle_{c} \varphi,
\end{aligned}
$$

where

$$
\triangle_{d}:=\mathbb{D} i v \overline{\mathbb{G}}_{1}, \quad \triangle_{c}:=\overline{\mathbb{C} u r l} \mathbb{G}^{\perp}
$$

are given. Since $\triangle_{c}: H_{P}^{o} \rightarrow H_{P}^{o}$ is invertible, the potential $\varphi$ is uniquely determined by $\omega$, while $\psi$ (and $u$ ) are given only up to a laminar flow $u_{\text {lam }}$ with vanishing $\mathbb{D} i v$ and $\overline{\mathbb{C} u r l}$. The laminar flow can be determined by boundary conditions where we have to take the mimetic Gaussian integral identity (2.3) into account. More precisely, we have the following proposition; cf. [36].

Proposition 2.2. The operator

$$
\mathcal{H}:=\left(\begin{array}{c}
\frac{\mathbb{D} i v}{\mathbb{C} u r l} \\
\mathbb{B}
\end{array}\right) \in \mathbb{R}^{\operatorname{dim} H_{S}+1, \operatorname{dim} H_{S}}
$$

provides a bijection between the spaces $H_{S}$ and

$$
V_{S}:=\left\{(\rho, \omega, \nu)^{T}: 1_{\operatorname{dim} H_{V}}^{T} \rho=1_{\operatorname{dim} H_{\partial V}}^{T} \nu\right\} .
$$


More precisely, if $(\rho, \omega, \nu)^{T}=\mathcal{H} u$, then $u=\mathcal{H}^{\dagger}(\rho, \omega, \nu)^{T}$; i.e., the inverse mapping on $V_{S}$ is given by the pseudoinverse $\mathcal{H}^{\dagger}:=\left(\mathcal{H}^{T} \mathcal{H}\right)^{-1} \mathcal{H}^{T}$ of $\mathcal{H}$.

In case of a solenoidal flow field from $\left\{u \in H_{S}: \mathbb{D} i v u=0\right\}$, we have by Proposition 2.1 that $\mathbb{D} i v \overline{\mathbb{G}}_{1} \psi=0$ and $\overline{\mathbb{C} u r l} \overline{\mathbb{G}}_{1} \psi=0$. Hence $\overline{\mathbb{G}}_{1} \psi=u_{\text {lam }}$ is a laminar flow and

$$
u=u_{\mathrm{lam}}+u_{\mathrm{sol}}=\overline{\mathbb{G}}_{1} \psi+\mathbb{G}^{\perp} \varphi, \quad \mathcal{H} u=(0,0, \nu)^{\mathrm{T}}+(0, \omega, 0)^{\mathrm{T}} .
$$

Further we have the following proposition.

Proposition 2.3. Every soleniodal flow field $u \in H_{S}$ can be written as

$$
u=\mathbb{G r a d}{ }^{\perp} \phi
$$

for some $\phi \in H_{P}$.

Proof. By properties of the tensor product it is easy to check that $\mathbb{D} i v \mathbb{G r a d} d^{\perp}=0$ so that the right-hand side of (2.6) is indeed solenoidal.

By Proposition 2.2 we obtain that $\operatorname{dim}\left(\left\{u \in H_{S}: \mathbb{D} i v u=0\right\}\right)=m n-1$. On the other hand, we have $\operatorname{dim}\left(\mathcal{R}\left(\mathbb{G r a d}{ }^{\perp}\right)\right)=\operatorname{dim} H_{P}-1=m n-1$.

Primal and dual TV/G norms. For our optical flow decomposition and estimation we need discrete versions of the TV norm $\|f\|_{\mathrm{TV}}:=\int|\nabla f| \mathrm{d} x \mathrm{~d} y$ and the G norm $\|f\|_{\mathrm{G}}:=\inf _{f=\operatorname{div} p}\||p|\|_{L_{\infty}}$, where $|p|:=\left(p_{1}^{2}+p_{2}^{2}\right)^{1 / 2}$ for a vector field $p=\left(p_{1}, p_{2}\right)$, $p_{1}, p_{2} \in L_{\infty}$. For a more sophisticated treatment of these norms in the continuous setting, see, e.g., [11, 21].

For $\omega \in H_{P}^{o}$ we set

$$
\|\omega\|_{\mathrm{TV}}:=|\mathbb{G} \omega|_{H_{E}^{o}} .
$$

This defines a seminorm on $H_{P}^{o}$. As usual we will skip the prefix "semi" in the following. Let $N:=\operatorname{dim} H_{P}^{o}$. According to the norm in $H_{E}^{o}$ let $S: \mathbb{R}^{\operatorname{dim} H_{E}^{o}} \rightarrow \mathbb{R}^{4 N}$ be the matrix which assigns the four appropriate elements of $H_{E}^{o}$ to each point of $H_{P}^{o}$; cf. the appendix. For $w:=\left(w^{1}, w^{2}, w^{3}, w^{4}\right)^{\mathrm{T}} \in \mathbb{R}^{4 N}$ with $w^{r}:=\left(w_{j}^{r}\right)_{j=1}^{N}, r=1, \ldots, 4$, we define the vector

$$
|w|:=\left(\left\|\left(w_{j}^{r}\right)_{r=1}^{4}\right\|_{2}\right)_{j=1}^{N} .
$$

Then the TV norm can be rewritten as

$$
\|\omega\|_{\mathrm{TV}}=\||S \mathbb{G} \omega|\|_{1} .
$$

Now the $\mathrm{G}$ norm of $\omega$ is defined by

$$
\|\omega\|_{\mathrm{G}}:=\min _{\omega=\mathbb{G}^{*} S^{*} w}\||w|\|_{\infty}=\min _{\omega=\overline{\mathbb{D} i v} S^{*} w}\||w|\|_{\infty} .
$$

Similarly, we can define the TV and the G norm on $H_{V}$. For $\rho \in H_{V}$ let

$$
\|\rho\|_{\overline{\mathrm{TV}}}:=\left|\overline{\mathbb{G}}_{2} \rho\right|_{H_{S}} .
$$

We focus on the second way of extending $\overline{\mathbb{G}}$ to $\overline{\mathbb{G}}_{2}$ and introduce the operator $\bar{S}$ : $\mathbb{R}^{\operatorname{dim} H_{S}^{o}} \rightarrow \mathbb{R}^{4 N}$ with $N:=\operatorname{dim} H_{V}$, which constantly extrapolates from $H_{S}^{o}$ to $H_{S}$ and assigns the elements of $H_{S}$ to those of $H_{V}$. For the matrix structure of $\bar{S}$, see the appendix. Then we obtain that

$$
\|\rho\|_{\mathrm{TV}}=\||\bar{S} \overline{\mathbb{G}} \rho|\|_{1}
$$


and

$$
\|\rho\|_{\overline{\mathrm{G}}}:=\min _{\rho=\overline{\mathbb{G}} * \bar{S}^{*} p}\||p|\|_{\infty}=\min _{\rho=\mathbb{D} i v \bar{S}^{*} p}\||p|\|_{\infty} .
$$

The G norm ( $\overline{\mathrm{G}}$ norm) can be considered as dual to the TV norm ( $\overline{\mathrm{TV}}$ norm) in the following sense.

Proposition 2.4. For $\tilde{\omega} \in \mathcal{R}\left(\mathbb{G}^{*} S^{*}\right)$ the following relation holds true:

$$
\sup _{\|\omega\|_{\mathrm{TV}} \neq 0} \frac{|\langle\tilde{\omega}, \omega\rangle|}{\|\omega\|_{\mathrm{TV}}}=\|\tilde{\omega}\|_{\mathrm{G}} .
$$

Proof. 1. Set

$$
L:=S \mathbb{G}: \mathbb{R}^{N} \rightarrow \mathbb{R}^{4 N}, \quad N:=\operatorname{dim} H_{E}^{o} .
$$

Then, using the notation (2.7), the relation (2.9) can be rewritten as

$$
\sup _{\||L \omega|\|_{1} \neq 0} \frac{|\langle\tilde{\omega}, \omega\rangle|}{\||L \omega|\|_{1}}=\min _{\tilde{\omega}=L^{\mathrm{T}} v}\||v|\|_{\infty}, \quad \tilde{\omega} \in \mathcal{R}\left(L^{\mathrm{T}}\right) .
$$

Let $\nu(\tilde{\omega}):=\sup _{\||L \omega|\|_{1} \neq 0} \frac{|\langle\tilde{\omega}, \omega\rangle|}{\||L \omega|\|_{1}}$. By applying the Schwarz inequality to the four parts of the corresponding vectors in $\mathbb{R}^{4 N}$, we obtain for all $v \in \mathbb{R}^{4 N}$ with $L^{\mathrm{T}} v=\tilde{\omega}$ that

$$
\langle\tilde{\omega}, \omega\rangle=\langle v, L \omega\rangle \leq\langle|v|,|L \omega|\rangle \quad \forall \omega \in \mathbb{R}^{N}
$$

and then obviously

$$
\langle|v|,|L \omega|\rangle \leq\||L \omega|\|_{1}\||v|\|_{\infty} \quad \forall \omega \in \mathbb{R}^{N} .
$$

Consequently, we have

$$
\nu(\tilde{\omega}) \leq \min _{\tilde{\omega}=L^{\mathrm{T}} v}\||v|\|_{\infty} .
$$

To show the reverse direction we consider the subspace $\mathcal{B}:=\mathcal{R}(L)$ of $\mathbb{R}^{4 N}$ equipped with the norm $\||\cdot|\|_{1}$. Let $\tilde{w}$ with $L^{\mathrm{T}} \tilde{w}=\tilde{\omega}$ be fixed. Then the mapping $l_{\tilde{w}}(L \omega):=\langle\tilde{w}, L \omega\rangle$ is a linear functional on $\mathcal{B}$ which has exactly the norm $\nu(\tilde{\omega})$. By the Hahn-Banach theorem this functional can be extended to a linear functional $l$ on $\left(\mathbb{R}^{4 N},\||\cdot|\|_{1}\right)$ with $\|l\|=\left\|l_{\tilde{w}}\right\|$. Then there exists $\tilde{v} \in \mathbb{R}^{4 N}$ such that $l(v)=\langle\tilde{v}, v\rangle$ for all $v \in \mathbb{R}^{4 N}$ and

$$
\langle\tilde{v}, L \omega\rangle=\langle\tilde{w}, L \omega\rangle \quad \forall \omega \in \mathbb{R}^{N} .
$$

Since this can be rewritten as

$$
\left\langle L^{\mathrm{T}} \tilde{v}, \omega\right\rangle=\left\langle L^{\mathrm{T}} \tilde{w}, \omega\right\rangle \quad \forall \omega \in \mathbb{R}^{N}
$$

the vector $\tilde{v}$ must fulfil $L^{\mathrm{T}} \tilde{v}=L^{\mathrm{T}} \tilde{w}=\tilde{\omega}$. Then

$$
\nu(\tilde{\omega})=\left\|l_{\tilde{w}}\right\|=\|l\|=\||\tilde{v}|\|_{\infty},
$$

where the last equality follows from the fact that $\||\cdot|\|_{1}$ and $\||\cdot|\|_{\infty}$ are dual norms on $\mathbb{R}^{4 N}$. Thus, $\nu(\tilde{\omega}) \geq \min _{\tilde{\omega}=L^{\mathrm{T}} v}\||v|\|_{\infty}$, and we obtain the assertion. 
3. Flow estimation. Assume now that we are given an image sequence $\{g(\cdot, j) \in$ $\left.H_{V}: j=1, \ldots, T\right\}$. For fixed $j \in \mathbb{N}$, we set $g_{t}=g_{t}(j):=g(\cdot, j+1)-g(\cdot, j)$. Then the data fitting functional (1.6) can be discretized by

$$
F(u)=\left\|\overline{\mathbb{G}}_{2} g \cdot u+g_{t}\right\|_{H_{V}}^{2}
$$

with the dotproduct on $H_{S}$ and by applying the mimetic Helmholtz decomposition (2.4) by

$$
F(\psi, \varphi)=\left\|\overline{\mathbb{G}}_{2} g \cdot \overline{\mathbb{G}}_{1} \psi+\overline{\mathbb{G}}_{2} g \cdot \mathbb{G}^{\perp} \varphi+g_{t}\right\|_{H_{V}}^{2} .
$$

This further decomposition can be useful from two points of view. First the vectors $\psi$ and $\varphi$ have only approximately half the lengths of the vector $u$. Second this approach allows for efficiently handling solenoidal flows, which are very common in computational fluid dynamics.

To discretize the boundary integral $\int_{\partial \Omega}\left(\partial_{\mathrm{n}} u\right)^{2} \mathrm{~d} s$ in (1.9) and (1.10) we use the operator

$$
\mathbb{B}^{\mathrm{n}}:=\left(\begin{array}{cc}
I_{n-1} \otimes B_{m}^{\mathrm{n}} & 0 \\
0 & B_{n}^{\mathrm{n}} \otimes I_{m-1}
\end{array}\right)
$$

with

$$
B_{m}^{\mathrm{n}}:=\left(\begin{array}{rrrrr}
-1 & 1 & \ldots & 0 & 0 \\
0 & 0 & \ldots & -1 & 1
\end{array}\right) \in \mathbb{R}^{2, m} .
$$

For the smoothing penalizer we distinguish between the linear and the TV approach.

Linear approach. The discrete version of the functional (1.9) becomes

$$
J(u)=F(u)+\lambda_{d}\left\|\overline{\mathbb{G}}_{2} \mathbb{D} i v u\right\|_{H_{S}}^{2}+\lambda_{c}\|\mathbb{G} \overline{\mathbb{C} u r l} u\|_{H_{E}^{o}}^{2}+\gamma\left\|\mathbb{B}^{\mathrm{n}} u\right\|_{\partial H_{S}}^{2} .
$$

The minimizer $u$ of (3.1) can be obtained by solving the corresponding normal equation.

Using the Helmholtz decomposition together with $u_{\left.\right|_{\partial H_{S}}}=\left(\overline{\mathbb{G}}_{1} \psi\right)_{\partial_{\partial H_{S}}}$ and (2.2), problem (3.1) can be rewritten as

$$
J(\psi, \varphi)=F(\psi, \varphi)+\lambda_{d}\left\|\overline{\mathbb{G}}_{2} \triangle_{d} \psi\right\|_{H_{S}}^{2}+\lambda_{c}\left\|\mathbb{G} \triangle_{c} \varphi\right\|_{H_{E}^{o}}^{2}+\gamma\left\|\mathbb{B}^{\mathrm{n}} \bar{G}_{1} \psi\right\|_{\partial H_{S}}^{2} .
$$

Since $\psi$ is determined only up to a constant, we add the additional condition

$$
1_{\operatorname{dim} H_{V+\partial V}}^{\mathrm{T}} \psi=0
$$

to make the functional strictly convex. This problem can be solved by subspace correction methods $[31,33]$. More precisely, we iterate the following process: we consider $\varphi$ as given and find the solution $\psi$ of (3.2) via the normal equation. Then we fix this solution as $\psi$ and compute $\varphi$.

In case of a solenoidal flow field $u \in H_{S}$ we can apply (2.5), and our variational problem reduces to

$$
\begin{aligned}
J(\psi, \varphi) & =F(\psi, \varphi)+\lambda_{c}\left\|\mathbb{G} \triangle_{c} \varphi\right\|_{H_{E}^{o}}^{2}+\gamma\left\|\mathbb{B}^{\mathrm{n}} \bar{G}_{1} \psi\right\|_{\partial H_{S}}^{2} \\
\text { s.t. } & \overline{\mathbb{G}}_{2} \triangle_{d} \psi=0, \quad 1_{\operatorname{dim} H_{V+\partial V}}^{\mathrm{T}} \psi=0 .
\end{aligned}
$$

The linear case was more extensively treated by some of the authors in [35]. 
$T V$ approach. Functional (1.10) becomes

$$
J(u)=\frac{1}{2} F(u)+\lambda_{d}\|\mathbb{D} i v u\|_{\overline{\mathrm{TV}}}+\lambda_{c}\|\overline{\mathbb{C} u r l} u\|_{\mathrm{TV}}+\frac{\gamma}{2}\left\|\mathbb{B}^{\mathrm{n}} u\right\|_{\partial H_{S}}^{2},
$$

and by applying the Helmholtz decomposition,

$$
J(\psi, \varphi)=\frac{1}{2} F(\psi, \varphi)+\lambda_{d}\left\|\triangle_{d} \psi\right\|_{\overline{\mathrm{TV}}}+\lambda_{c}\left\|\triangle_{c} \varphi\right\|_{\mathrm{TV}}+\frac{\gamma}{2}\left\|\mathbb{B}^{\mathrm{n}} \bar{G}_{1} \psi\right\|_{\partial H_{S}}^{2},
$$

where $1_{\operatorname{dim} H_{V+\partial V}}^{\mathrm{T}} \psi=0$. In case of solenoidal flows the functional further reduces as in the linear case.

To solve (3.3) we propose using SOCP; cf. section 6 .

Alternatively, we can again apply a splitting algorithm in (3.4) which iteratively fixes $\varphi$ as previously computed and finds the minimizer $\psi$ and conversely. Then any of the subproblems with fixed $\varphi$ or fixed $\psi$ can be written in the form

$$
\frac{1}{2}\|A x-b\|_{2}^{2}+\lambda\||L x|\|_{1},
$$

where $A: \mathbb{R}^{\operatorname{dim} H_{V+\partial V}} \rightarrow \mathbb{R}^{\operatorname{dim} H_{V}}, L: \mathbb{R}^{\operatorname{dim} H_{V+\partial V}} \rightarrow \mathbb{R}^{4 \operatorname{dim} H_{V}}$ in case of fixed $\varphi$ and $A: \mathbb{R}^{\operatorname{dim} H_{P}^{o}} \rightarrow \mathbb{R}^{\operatorname{dim} H_{V}}, L: \mathbb{R}^{\operatorname{dim} H_{P}^{o}} \rightarrow \mathbb{R}^{4 \operatorname{dim} H_{P}^{o}}$ in case of fixed $\psi$. The minimizer of (3.5) can be found by applying dual optimization techniques as proposed by Chambolle and coworkers $[3,7,6]$.

4. Flow decomposition. In this section, we want to decompose a given flow vector $u \in H_{S}$ in a meaningful way. To this end we have to compute some basic decomposition of $u$ first. We apply Proposition 2.2 and consider $\mathcal{H} u=(\rho, \omega, \nu)^{\mathrm{T}}$. Let

$$
c_{\rho}:=\frac{1_{\operatorname{dim} H_{V}}^{\mathrm{T}} \rho}{\operatorname{dim} H_{V}}, \quad c_{\omega}:=\frac{1_{\operatorname{dim} H_{P}^{o}}^{\mathrm{T}} \omega}{\operatorname{dim} H_{P}^{o}}
$$

be the discrete versions of $|\Omega|^{-1} \int_{\Omega} \operatorname{div} u \mathrm{~d} x \mathrm{~d} y$ and $|\Omega|^{-1} \int_{\Omega} \operatorname{curl} u \mathrm{~d} x \mathrm{~d} y$ and

$$
\rho_{\text {const }}:=c_{\rho} 1_{\operatorname{dim} H_{V}}, \quad \omega_{\text {const }}:=c_{\omega} 1_{\operatorname{dim} H_{P}^{o}} .
$$

Then we can decompose $(\rho, \omega, \nu)^{\mathrm{T}} \in V_{S}$ as

$$
(\rho, \omega, \nu)=\left(\rho_{\text {const }}, \omega_{\text {const }}, \nu\right)+\left(\rho_{\text {var }}, \omega_{\text {var }}, 0\right),
$$

where $1_{\operatorname{dim} H_{V}}^{\mathrm{T}} \rho_{\mathrm{var}}=1_{\operatorname{dim} H_{P}^{o}}^{\mathrm{T}} \omega_{\mathrm{var}}=0$. Obviously, both summands are in $V_{S}$ again, so that

$$
u=u_{\text {const }}+u_{\mathrm{var}}
$$

is the corresponding basic decomposition of $u \in H_{S}$, where

$$
u_{\text {const }}=\mathcal{H}^{\dagger}\left(\left(\rho_{\text {const }}, \omega_{\text {const }}, \nu\right)^{\mathrm{T}}, \quad u_{\text {var }}=\mathcal{H}^{\dagger}\left(\rho_{\text {var }}, \omega_{\text {var }}, 0\right)^{\mathrm{T}} .\right.
$$

We call vector $u_{\text {const }}$ (resp., $\left.\left(\rho_{\text {const }}, \omega_{\text {const }}, \nu\right)\right)$ the basic pattern of the nonrigid flow and its boundary behavior, while $u_{\mathrm{var}}$ (resp., $\left.\left(\rho_{\mathrm{var}}, \omega_{\mathrm{var}}, 0\right)\right)$ is related to the variable (oscillating) flow pattern.

We are interested in further decomposing the intrinsic flow variation $u_{\text {var }}$ into a structural part $u^{s}$ and a texture part $u^{t}$, i.e.,

$$
u_{\mathrm{var}}=u^{s}+u^{t} .
$$


This can be done in two ways. The first approach uses Proposition 2.2. Given $u \in H_{S}$, we compute $(\rho, \omega, \nu)^{\mathrm{T}}=\mathcal{H} u$ and then $\rho_{\text {var }}=\rho-\rho_{\text {const }}$ and $\omega_{\text {var }}=\omega-\omega_{\text {const }}$ by (4.1). Next we decompose $\rho_{\text {var }}=\rho^{s}+\rho^{t}$ and $\omega_{\text {var }}=\omega^{s}+\omega^{t}$ by minimizing separately

$$
\begin{aligned}
J\left(\rho^{s}, \rho^{t}\right) & =\frac{1}{2}\left\|\rho_{\mathrm{var}}-\rho^{s}-\rho^{t}\right\|_{2}^{2}+\lambda_{d}\left\|\rho^{s}\right\|_{\overline{\mathrm{TV}}}+\mu_{d}\left\|\rho^{t}\right\|_{\overline{\mathrm{G}}}, \\
J\left(\omega^{s}, \omega^{t}\right) & =\frac{1}{2}\left\|\omega_{\mathrm{var}}-\omega^{s}-\omega^{t}\right\|_{2}^{2}+\lambda_{c}\left\|\omega^{s}\right\|_{\mathrm{TV}}+\mu_{c}\left\|\omega^{t}\right\|_{\mathrm{G}} .
\end{aligned}
$$

The minimizers can be computed as proposed in [3]. By (2.8) we see that $\rho^{t} \in$ $\mathcal{R}\left(\overline{\mathbb{G}}^{*}\right)=\mathcal{R}\left(\mathbb{D} i v_{\left.\right|_{H_{S}^{o}}}\right)$, and since $1^{\mathrm{T}} \mathbb{D} i v_{H_{H_{S}^{o}}}=0$ we have that $\left(\rho^{t}, \omega^{t}, 0\right)^{\mathrm{T}} \in V_{S}$ and further by (4.2) that $\left(\rho^{s}, \omega^{s}, 0\right)^{\mathrm{T}} \in V_{S}$. Thus we can finally compute $u^{s}$ and $u^{t}$ by

$$
u^{s}=\mathcal{H}^{\dagger}\left(\rho^{s}, \omega^{s}, 0\right)^{\mathrm{T}}, \quad u^{t}=\mathcal{H}^{\dagger}\left(\rho^{t}, \omega^{t}, 0\right)^{\mathrm{T}} .
$$

In this paper, we prefer a second approach that computes the components of $u$ directly. This variational approach extends Meyer's model for the decomposition of scalar-valued functions to the simultaneous decomposition of vector fields into basic and variable (structural and textural) flow patterns. Moreover, it also fits into our flow estimation-decomposition model in the next section. For $u \in H_{S}$ we propose to to find $u_{\text {const }} \in H_{S}$ and $u^{s}, u^{t} \in H_{S}^{o}$ by minimizing

$$
\begin{aligned}
& J\left(u_{\text {const }}, u^{s}, u^{t}\right)=\lambda_{d}\left\|\mathbb{D} i v u^{s}\right\|_{\overline{\mathrm{TV}}}+\lambda_{c}\left\|\overline{\mathbb{C} u r l} u^{s}\right\|_{\mathrm{TV}} \\
& \text { s.t. }\left\|\mathbb{D} i v u^{t}\right\|_{\overline{\mathrm{G}}} \leq \delta_{d}, \quad\left\|\overline{\mathbb{C} u r l} u^{t}\right\|_{\mathrm{G}} \leq \delta_{c}, \\
& u_{\text {const }}+u^{s}+u^{t}=u, \\
& \overline{\mathbb{G}} \mathbb{D} i v u_{\text {const }}=0, \quad \mathbb{G} \overline{\mathbb{C} u r l} u_{\text {const }}=0, \\
& 1_{\mathrm{dim} H_{P}^{o}}^{\mathrm{T}} \overline{\mathbb{C} u r l} u^{s}=0 .
\end{aligned}
$$

Concerning the last three constraints we note the following: to obtain the desired decomposition we have to ensure that $\mathbb{D} i v u_{\text {const }}$ and $\overline{\mathbb{C} u r l} u_{\text {const }}$ are constant vectors $c 1$ and that $1_{\operatorname{dim} H_{V}}^{\mathrm{T}} \mathbb{D} i v\left(u^{s}+u^{t}\right)=0,1_{\operatorname{dim} H_{P}^{o}}^{\mathrm{T}} \overline{\mathbb{C} u r l}\left(u^{s}+u^{t}\right)=0$. The first two conditions are fulfilled by the fourth and fifth constraint. The third condition is fulfilled by the mimetic Gaussian integral identity and since $u^{s}, u^{t} \in H_{S}^{o}$. The last condition follows by the last constraint and since $\overline{\mathbb{C} u r l} u^{t} \in \mathcal{R}(\overline{\mathbb{D} i v})$ and $1^{\mathrm{T}} \overline{\mathbb{D} i v}=0$.

The solution of (4.3) can be found by SOCP, as shown in section 6 .

5. Flow estimation and decomposition. In this section, we combine optical flow estimation with structure-texture flow decomposition. Given image sequence $\left\{g(\cdot, j) \in H_{V}: j=1, \ldots, T\right\}$, we want to compute the components $u_{\text {const }}$ with constant divergence and curl, the large scale patterns $u^{s} \in H_{S}^{o}$ of divergence and curl with bounded TV norms, and the small scale patterns $u^{t} \in H_{S}^{o}$ of divergence and curl with bounded $\mathrm{G}$ norms. To this end, we introduce the fitting functional

$$
F\left(u_{\text {const }}, u^{s}, u^{t}\right)=\left\|\overline{\mathbb{G}}_{2} g \cdot\left(u_{\text {const }}+u^{s}+u^{t}\right)+g_{t}\right\|_{2}^{2} .
$$

Then, with respect to the previous section, one can consider minimizing

$$
\begin{aligned}
J\left(u_{\text {const }}, u^{s}, u^{t}\right) & =\frac{1}{2} F\left(u_{\text {const }}, u^{s}, u^{t}\right)+\lambda_{d}\left\|\mathbb{D} i v u^{s}\right\|_{\overline{\mathrm{TV}}}+\lambda_{c}\left\|\overline{\mathbb{C} u r l} u^{s}\right\|_{\mathrm{TV}}+\frac{\gamma}{2}\left\|\mathbb{B}^{n} u\right\|_{\partial H_{S}}^{2} \\
\text { s.t. } & \left\|\mathbb{D} i v u^{t}\right\|_{\overline{\mathrm{G}}} \leq \delta_{d}, \quad\left\|\overline{\mathbb{C} u r l} u^{t}\right\|_{\mathrm{G}} \leq \delta_{c}, \\
& \overline{\mathbb{G}} \mathbb{D} i v u_{\text {const }}=0, \quad \mathbb{G} \overline{\mathbb{C} u r l} u_{\text {const }}=0, \\
& 1_{\mathrm{dim} H_{P}^{o}}^{\mathrm{T}} \overline{\mathbb{C} u r l} u^{s}=0 .
\end{aligned}
$$


Unfortunately, this approach is not well posed. For the image areas where $\nabla g=0$, the data term disappears, and the local constraints on the $\mathrm{G}$ norms will lead to unbounded solutions. Therefore, we propose to replace the $\mathrm{G}$ norm by the $L_{2}$ norm, which leads to

$$
\begin{aligned}
& J\left(u_{\text {const }}, u^{s}, u^{t}\right)= \frac{1}{2} F\left(u_{\text {const }}, u^{s}, u^{t}\right)+\lambda_{d}\left\|\mathbb{D} i v u^{s}\right\|_{\overline{\mathrm{TV}}}+\lambda_{c}\left\|\overline{\mathbb{C} u r l} u^{s}\right\|_{\mathrm{TV}} \\
& \quad+\mu_{d}\left\|\mathbb{D} i v u^{t}\right\|_{2}^{2}+\mu_{c}\left\|\overline{\mathbb{C} u r l} u^{t}\right\|_{2}^{2}+\frac{\gamma}{2}\left\|\mathbb{B}^{n} u\right\|_{\partial H_{S}}^{2}, \\
& \text { s.t. } \quad \overline{\mathbb{G}} \mathbb{D} i v u_{\text {const }}=0, \quad \overline{\mathbb{G} u r l} u_{\text {const }}=0, \\
& 1_{\operatorname{dim} H_{P}^{o}}^{\mathrm{T}}\left(\overline{\mathbb{C} u r l} u^{s}+\overline{\mathbb{C} u r l} u^{t}\right)=0 .
\end{aligned}
$$

We will see in our experiments that this approach works well, although the superiority of the $\mathrm{G}$ norm over the $L_{2}$ norm in capturing (scalar) oscillating patterns was experimentally shown in [4].

Finally, we are interested in solenoidal flows $u \in H_{S}$. Coupling the mimetic Helmholtz decomposition and the basic decomposition, we obtain with the laminar flow $\overline{\mathbb{G}}_{1} \psi$ and $\varphi=\varphi_{\text {const }}+\varphi^{s}+\varphi^{t}$ that

$$
u=u_{\text {const }}+u^{s}+u^{t}=\left(\overline{\mathbb{G}}_{1} \psi+\mathbb{G}^{\perp} \varphi_{\text {const }}\right)+\mathbb{G}^{\perp} \varphi^{s}+\mathbb{G}^{\perp} \varphi^{t} .
$$

By Proposition 2.3 and since $\left.\mathbb{G r a d}\right|_{\left.\right|_{H_{P}^{o}}} ^{\perp}=\mathbb{G}^{\perp}$ this can be rewritten as

$$
u=\mathbb{G r a d} d^{\perp}\left(\phi_{\text {const }}+\varphi^{s}+\varphi^{t}\right), \quad \phi \in H_{P}, \varphi^{s}, \varphi^{t} \in H_{P}^{o}
$$

Then the fitting term reads

$$
F\left(\phi_{\text {const }}, \varphi^{s}, \varphi^{t}\right)=\left\|\overline{\mathbb{G}}_{2} g \cdot \mathbb{G r a d}{ }^{\perp}\left(\phi_{\text {const }}+\varphi^{s}+\varphi^{t}\right)+g_{t}\right\|_{2}^{2},
$$

and (5.1) can be rewritten as

$$
\begin{aligned}
J\left(\phi_{\text {const }}, \varphi^{s}, \varphi^{t}\right)= & \frac{1}{2} F\left(\phi_{\text {const }}, \varphi^{s}, \varphi^{t}\right)+\lambda_{c}\left\|\triangle_{c} \varphi^{s}\right\|_{\mathrm{TV}}+\mu_{c}\left\|\triangle_{c} \varphi^{t}\right\|_{2}^{2} \\
& +\frac{\gamma}{2}\left\|\mathbb{B}^{n} \mathbb{G} r a d^{\perp} \phi_{\text {const }}\right\|_{\partial H_{S}}^{2} \\
\text { s.t. } \quad & \mathbb{G} \frac{2}{\mathbb{C} u r l} \mathbb{G} r a d^{\perp} \phi_{\text {const }}=0,1_{\operatorname{dim} H_{P}^{o}}^{\mathrm{T}} \triangle_{c}\left(\varphi^{s}+\varphi^{t}\right)=0, \\
& 1_{\text {dim } H_{P}}^{\mathrm{T}} \phi_{\text {const }}=0,
\end{aligned}
$$

where the last constraint appears since $\phi_{\text {const }}$ is determined only up to an additive element from the kernel of $\mathbb{G} r a d^{\perp}$, which consists of constant vector fields.

6. Algorithmic aspect-SOCP. Our computational approach to flow estimation via (4.3) and to simultaneous flow estimation and decomposition via (5.2) is based on SOCP [20]. This amounts to minimizing a linear objective function subject to the constraints that several affine functions of the variables have to lie in a second-order cone $\mathcal{L}^{n+1} \subset \mathbb{R}^{n+1}$ defined by the convex set

$$
\mathcal{L}^{n+1}=\left\{\left(\begin{array}{l}
x \\
t
\end{array}\right)=\left(x_{1}, \ldots, x_{n}, t\right)^{\top}:\|x\|_{2} \leq t\right\} .
$$

With this notation, the general form of an SOCP is given by

$$
\inf _{x \in \mathbb{R}^{n}} f^{\top} x, \quad \text { s.t. }\left(\begin{array}{c}
A_{i} x+b_{i} \\
c_{i}^{T} x+d_{i}
\end{array}\right) \in \mathcal{L}^{n+1}, \quad i=1, \ldots, r .
$$


Problem (6.1) is a convex program for which efficient large-scale solvers are available [22]. In connection with TV-based image decomposition the application of SOCPs was recently suggested in $[12,34]$.

We reformulate the variational approach (4.3) as the SOCP

$$
\begin{gathered}
J\left(u_{\text {const }}, u^{s}, u^{t}\right)=\lambda_{d} 1_{\operatorname{dim} H_{V}}^{\mathrm{T}} v+\lambda_{c} 1_{\operatorname{dim} H_{P}^{\circ}}^{\mathrm{T}} w, \\
\text { s.t. } u_{\text {const }}+u^{s}+u^{t}=u, \quad \overline{\mathbb{G}} \mathbb{D} i v u_{\text {const }}=0, \quad \bar{G} \overline{\mathbb{C} u r l} u_{\text {const }}=0, \\
1_{\operatorname{dim} H_{P}^{o}}^{\overline{\mathbb{C}} u r l} u^{s}=0, \quad \mathbb{D} i v u^{t}=\mathbb{D} i v p_{d}, \quad \overline{\mathbb{C} u r l} u^{t}=\mathbb{D i v} p_{c}, \\
\left(\begin{array}{c}
\left(\overline{\mathbb{G}} \mathbb{D} i v u^{s}\right)_{V_{i+\frac{1}{2}, j+\frac{1}{2}}} \\
v_{V_{i+\frac{1}{2}, j+\frac{1}{2}}}
\end{array}\right) \in \mathcal{L}^{5}, \quad\left(\begin{array}{c}
\left(\overline{\mathbb{G} \mathbb{C} u r l} u^{s}\right)_{P_{i, j}} \\
w_{P_{i, j}}
\end{array}\right) \in \mathcal{L}^{5}, \\
\left(\begin{array}{c}
\left(p_{d}\right)_{V_{i+\frac{1}{2}, j+\frac{1}{2}}} \\
\delta_{d}
\end{array}\right) \in \mathcal{L}^{5}, \quad\left(\begin{array}{c}
\left(p_{c}\right)_{P_{i, j}} \\
\delta_{c}
\end{array}\right) \in \mathcal{L}^{5} .
\end{gathered}
$$

In order to incorporate the quadratic terms of the variational approaches to optical flow estimation, we use the following rotated version of the standard cone:

$$
\mathcal{R}^{n+2}:=\left\{\left(x, x_{n+1}, x_{n+2}\right)^{\top} \in \mathbb{R}^{n+2}: \frac{1}{2}\|x\|_{2}^{2} \leq x_{n+1} x_{n+2}, x_{n+1}, x_{n+2} \geq 0\right\}
$$

Fixing $x_{n+2}=1 / 2$, we have $\|x\|_{2}^{2} \leq x_{n+1}$. Now we can rewrite (5.2) as follows:

$$
\begin{aligned}
& J\left(\phi_{\text {const }}, \varphi^{s}, \varphi^{t}\right)=v+\mu_{c} t+\lambda_{c} 1_{\operatorname{dim} H_{P}^{o}}^{\mathrm{T}} w, \\
& \text { s.t. } \mathbb{G} \overline{\mathbb{C} u r l} \mathbb{G r a d}{ }^{\perp} \phi_{\text {const }}=0, \quad 1_{\operatorname{dim} H_{P}}^{\mathrm{T}} \phi_{\text {const }}=0 \text {, } \\
& 1_{\operatorname{dim} H_{P}^{o}}^{\mathrm{T}} \triangle_{c}\left(\varphi^{s}+\varphi^{t}\right)=0, \\
& \left(\begin{array}{c}
\left(\overline{\mathbb{G}} \triangle_{c} \varphi^{s}\right)_{V_{i+\frac{1}{2}, j+\frac{1}{2}}} \\
w_{V_{i, j}}
\end{array}\right) \in \mathcal{L}^{5}, \quad\left(\begin{array}{c}
\triangle_{c} \varphi^{t} \\
t \\
1 / 2
\end{array}\right) \in \mathcal{R}^{\operatorname{dim} H_{P}+2}, \\
& \left(\begin{array}{c}
\overline{\mathbb{G}}_{2} g \cdot \mathbb{G r a d} \\
\operatorname{Gr}^{\perp}\left(\phi_{\text {const }}+\varphi^{s}+\varphi^{t}\right)+g_{t} \\
v \\
1 / 2
\end{array}\right) \in \mathcal{R}^{\operatorname{dim} H_{V}+2} .
\end{aligned}
$$

7. Numerical examples. In this section we verify our approaches by numerical examples. The programs were written in MATLAB and used the software package SeDuMi for SOCP [27]. Unfortunately, we do not have an automatized choice of parameter values. Parameter values were chosen by hand following two general rules: (i) choose the weights as small as possible in order to not smooth out turbulent motion, (ii) for TV terms, smaller values than in the linear case (e.g., (21)) are appropriate, because these regularizers return larger values than their linear counterparts (assuming that image data are scaled to the range $[0,1]$ ). Rule (ii) leads to parameter values of the order $\propto e^{-4}$. Thanks to the mimetic discretization, this suffices for numerical stability

Flow estimation. We start by comparing flow estimations obtained by the linear approach (3.2) and the TV approach (3.4). We consider the artificial example in Figure 7.1 with the groundtruth on top of Figure 7.2. Figure 7.2 (middle) was obtained by solving the linear systems of equations resulting from (3.2) with parameters 

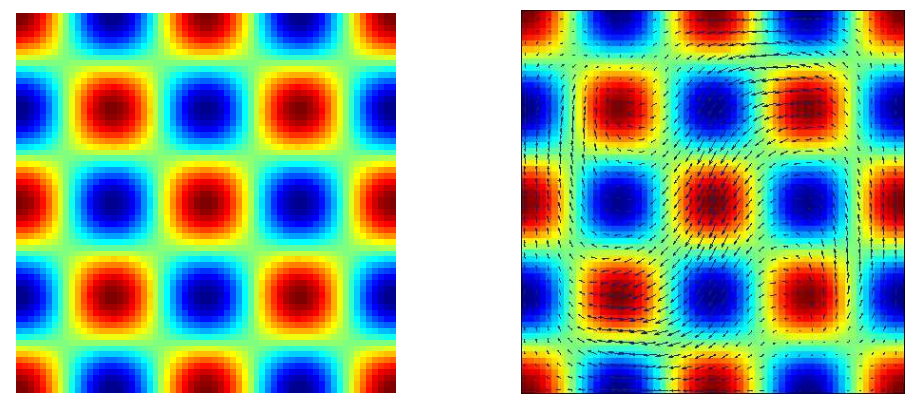

FIG. 7.1. Frame $g$ (left) and its warping with the groundtruth flow (right) for flow estimation by the linear approach and the TV approach.
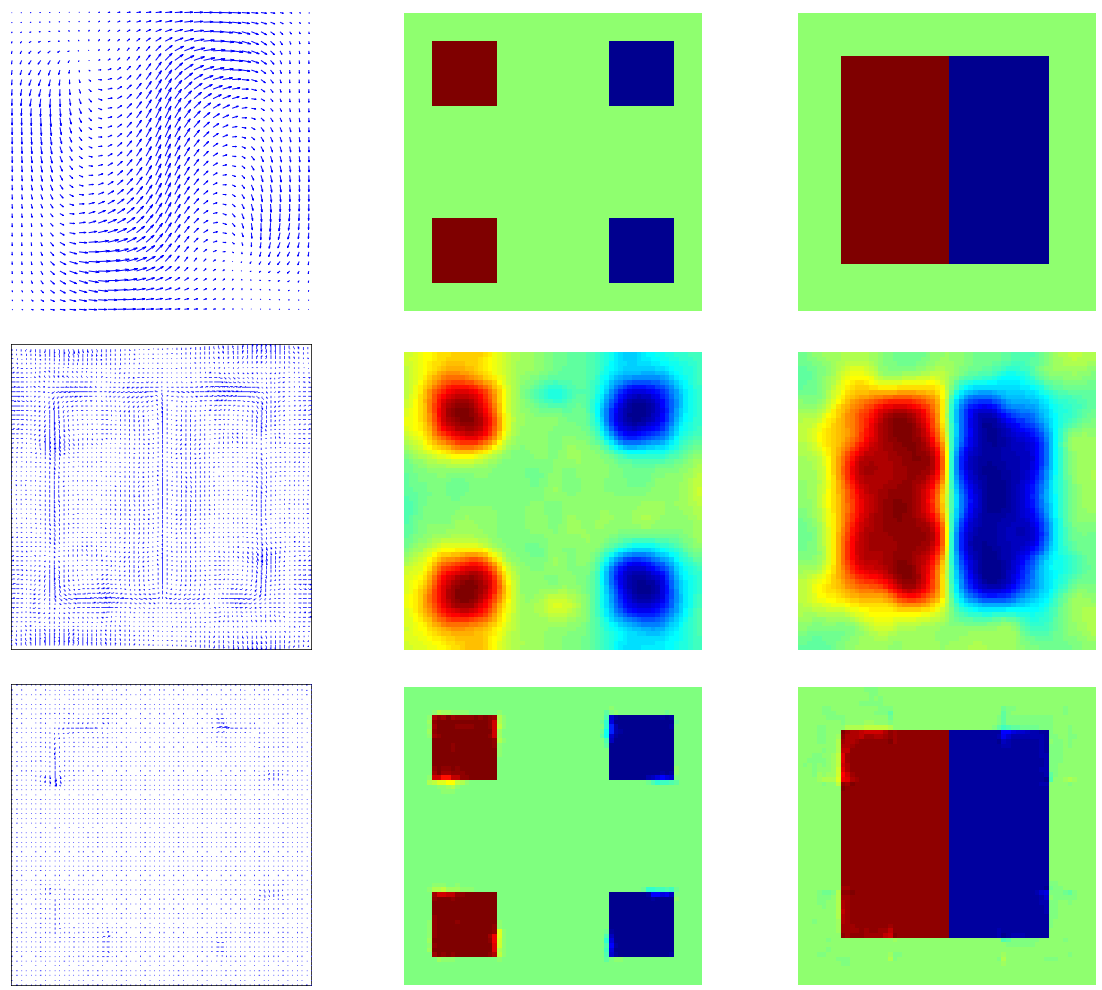

FIG. 7.2. Top: The groundtruth flow u, its div and curl (left to right). Middle: Linear reconstruction by (3.1), difference between $u$ and its estimation, reconstructed div and curl (left to right). Bottom: TV reconstruction by (3.3), difference between $u$ and its estimation, reconstructed div and curl (left to right).

$\lambda_{d}=0.06, \lambda_{c}=0.048$. As the boundary parameter we have used $\gamma=0.04$. The result shows the typical blurring effects at the edges. If we decrease the parameter values, the rectangular shape of div and curl becomes easier to see, but the artifacts always visible in Figure 7.2 (middle) increase too. The bottom of Figure 7.2 contains the solution of (3.3) with the parameters $\lambda_{d}=e^{-6}, \lambda_{c}=e^{-6}$, and $\gamma=e^{-5}$ by SOCP and 17 iterations. As expected for this example, the TV approach gives very good results by preserving discontinuities of the flow derivatives (div, curl). 
Flow decomposition. Figure 7.3 shows a turbulent flow field $u$ as groundtruth along with its divergence $\rho$ and curl $\omega$. Applying the variational method (4.3) with $\lambda_{d}=0.2, \lambda_{c}=0.5$, and $\delta_{d}=0.05, \delta_{c}=0.1$, we obtain the decompositions depicted in Figures 7.4 and 7.5. The structural and textural components recovered the interesting motion patterns at different scales, which are not easily visible in the flow $u$ itself.
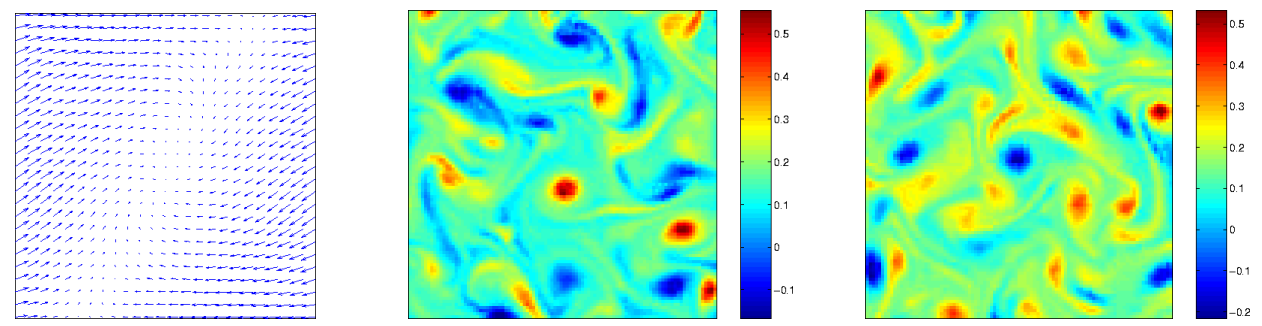

FIG. 7.3. Groundtruth data to be decomposed: flow field u (left), its divergence field $\rho$ (middle), and its curl field $\omega$ (right).
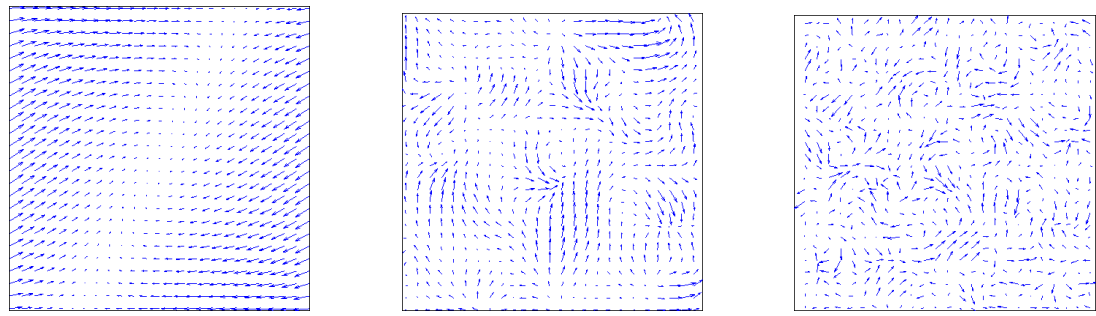

FIG. 7.4. The components of the flow $u$ from Figure 7.3: $u_{\text {const }}$ (left), $u^{s}$ (middle), and $u^{t}$ (right). The vectors of $u^{s}, u^{t}$ are scaled up for better visibility. Note that despite $|u| \approx\left|u_{\text {const }}\right|$, the structural and texture parts $u^{s}$ and $u^{t}$ are recovered well.
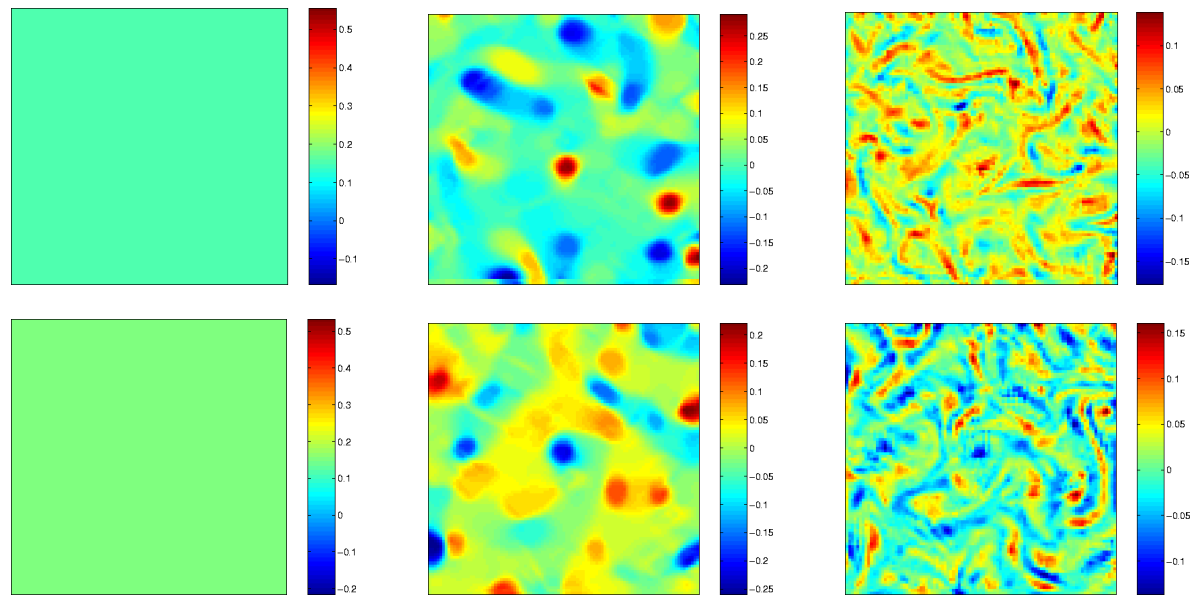

FIG. 7.5. Decomposition of $u$ from Figure 7.3 with the approach (4.3). Top: $\rho_{\text {const }}, \rho^{s}, \rho^{t}$ (left to right). Bottom: $\omega_{\text {const }}, \omega^{s}, \omega^{t}$ (left to right). The structure and texture components reveal turbulent flow patterns at different scales, which are not easily visible in the flow $u$ itself. 

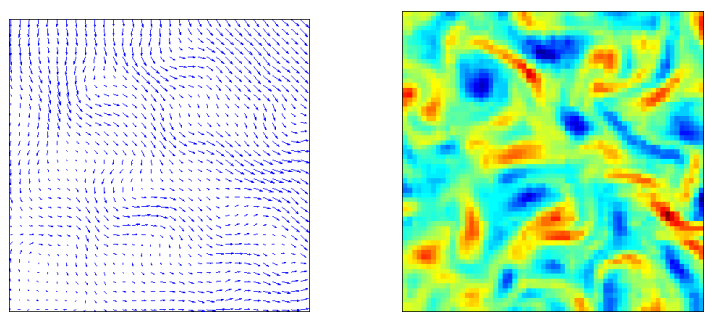

FIG. 7.6. Groundtruth data u (left) and its curl (right) to be estimated from a corresponding artificially created image sequence. Here $u$ is a superposition of a laminar flow and turbulent vortices.
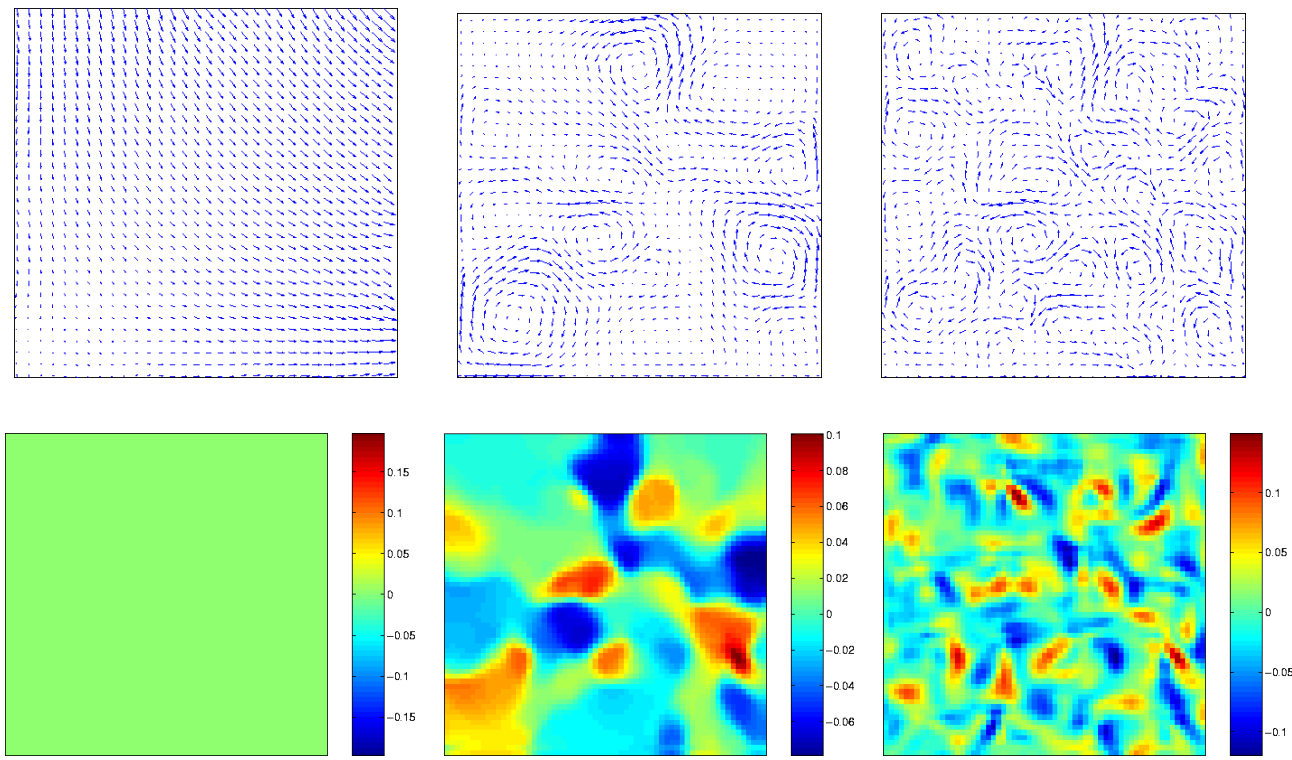

FIG. 7.7. Estimated and decomposed flow corresponding to Figure 7.6 using the $T V-L_{2}$ approach (5.2). Top: $u_{\text {const }}, u^{s}$, and $u^{t}$ (left to right). Bottom: $\omega_{\text {const }}, \omega^{s}$, and $\omega^{t}$ (left to right).
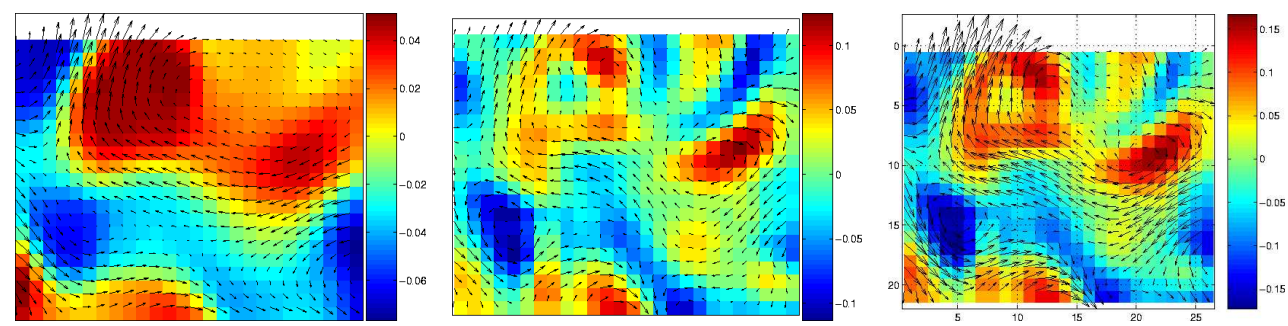

FIG. 7.8. Close-up view of a section of Figure 7.7. From left to right: $\omega^{s}, \omega^{t}, \omega^{s}+\omega^{t}$ with the corresponding flows as overlays.

Flow estimation and decomposition. In this section we will validate the flow estimation-decomposition model (5.2). First we create a divergence-free groundtruth flow field $u$ by superimposing a dominant laminar flow with some turbulent vortex structures; see Figure 7.6. Using this flow, an artificial image sequence $\{g\}$ was created.

Figures 7.7 and 7.8 show the decomposition-based optical flow estimates, where 

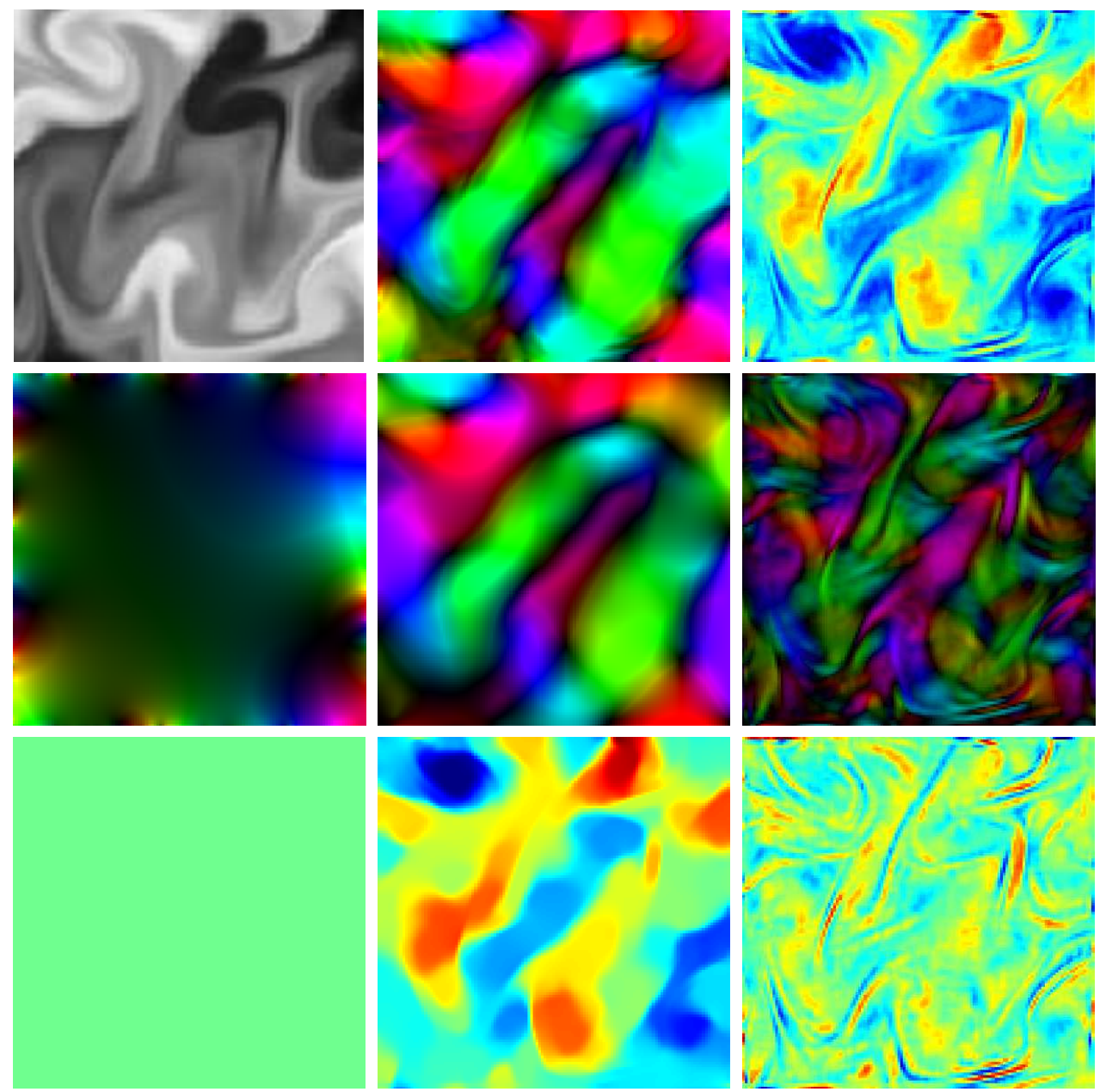

FIG. 7.9. Top-left: frame of a real image sequence depicting the mixture of fluorescein and water [18]. Top-center: the turbulent solenoidal flow estimated by minimizing (5.2). Flow vectors are color-coded for better visibility (color $\simeq$ direction, magnitude $\simeq$ brightness). Top-right: the curl field comprising large-scale patterns immersed in turbulent oscillations. Middle, from left to right: $u_{\text {const }}, u^{s}, u^{t}$. Note that $u_{\text {const }}$ contains the (nonvanishing) boundary values, and that $u^{s}$ and $u^{t}$ separate coherent motion patterns and turbulent fluctuations, respectively. Bottom, from left to right: the curl fields $\omega_{\text {const }}, \omega^{s}$, and $\omega^{t}$.

we have used $\lambda_{c}=6 e^{-5}$ and $\mu_{c}=3 e^{-4}$. The boundary parameter was chosen slightly smaller than $\lambda_{d}$. The $u_{\text {const }}$ component nicely recovers the laminar flow, whereas the structural and textural components reveal the turbulent curl field. Finally, Figure 7.8 gives a close-up view of a section of Figure 7.7.

A real-world example. Figure 7.9 (top-left) shows a sample image of the experimental evaluation of the spreading of a low-diffusivity dye in a two-dimensional turbulent flow, forced at a large scale. The passive scalar is a mixture of fluorescein and water. The divergence of the corresponding flow vanishes. For more details about the experimental setup, we refer to [18].

Figure 7.9 shows the components of the flow and curl field estimated in terms of $\phi_{\text {const }}, \varphi^{s}, \varphi^{t}$ by minimizing (5.2) (parameter values: $\lambda_{c}=1 e^{-4}, \mu_{c}=3 e^{-4}, \gamma=$ $\left.5 e^{-4}\right)$. This result clearly demonstrates how the convex constrained optimization 
approach allows for estimating complex flows while simultaneously separating largescale coherent motion patterns from turbulent fluctuations.

Appendix. Additional matrix notation. The extensions $\overline{\mathbb{G}}_{1}, \overline{\mathbb{G}}_{2}$ of $\overline{\mathbb{G}}=$ $-\left(\mathbb{D} i v_{\left.\right|_{H_{S}^{o}}}\right)^{*}=\left(\begin{array}{c}I_{n-1} \otimes D_{m-1} \\ D_{n-1} \otimes I_{m-1}\end{array}\right)$ are given as follows. Let

$$
D_{m}^{1}:=\left(\begin{array}{rrrrrrr}
-2 & 2 & 0 & \ldots & 0 & 0 & 0 \\
0 & -1 & 1 & \ldots & 0 & 0 & 0 \\
& & \ddots & \ddots & \ddots & & \\
0 & 0 & 0 & \ldots & -1 & 1 & 0 \\
0 & 0 & 0 & \ldots & 0 & -2 & 2
\end{array}\right) \in \mathbb{R}^{m, m+1}
$$

and

$$
D_{m}^{2}:=\left(\begin{array}{rrrrrrr}
-1 & 1 & 0 & \ldots & 0 & 0 & 0 \\
-1 & 1 & 0 & \ldots & 0 & 0 & 0 \\
0 & -1 & 1 & \ldots & 0 & 0 & 0 \\
& & \ddots & \ddots & \ddots & & \\
0 & 0 & 0 & \ldots & -1 & 1 & 0 \\
0 & 0 & 0 & \ldots & 0 & -1 & 1 \\
0 & 0 & 0 & \ldots & 0 & -1 & 1
\end{array}\right) \in \mathbb{R}^{m+1, m}
$$

and let the diagonal matrices $Z_{1}$ and $Z_{2}$ be defined by

$$
\begin{gathered}
Z_{1}:=\left(0_{(n-1) m, m-1}, I_{(n-1)(m+1)}, 0_{(n-1) m, m-1}\right) \\
Z_{2}:=\left(\begin{array}{ccc}
I_{m-1} & 0_{m-1,(n-1)(m+1)} & 0_{m-1, m-1} \\
0_{(n-1)(m-1), m-1} & I_{n-1} \otimes\left(0_{m-1,1}, I_{m-1}, 0_{m-1,1}\right) & 0_{(n-1)(m-1), m-1} \\
0_{m-1, m-1} & 0_{m-1,(n-1)(m+1)} & I_{m-1}
\end{array}\right),
\end{gathered}
$$

where $0_{m, n}$ is the matrix consisting of $m \times n$ zeros. Then

$$
\overline{\mathbb{G}}_{1}:=\left(\begin{array}{c}
\left(I_{n-1} \otimes D_{m}^{1}\right) Z_{1} \\
\left(D_{n}^{1} \otimes I_{m-1}\right) Z_{2}
\end{array}\right), \quad \overline{\mathbb{G}}_{2}:=\left(\begin{array}{c}
I_{n-1} \otimes D_{m-1}^{2} \\
D_{n-1}^{2} \otimes I_{m-1}
\end{array}\right) .
$$

The matrices $S$ and $\bar{S}$ for the computation of the TV and G norm can be defined using

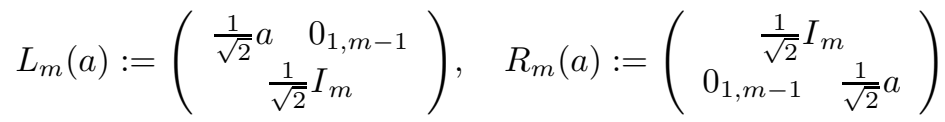

as

$$
\begin{aligned}
S & :=\left(\begin{array}{cc}
I_{n-2} \otimes L_{m-3}(0) & 0 \\
I_{n-2} \otimes R_{m-3}(0) & 0 \\
0 & L_{n-3}(0) \otimes I_{m-2} \\
0 & R_{n-3}(0) \otimes I_{m-2}
\end{array}\right), \\
\bar{S} & :=\left(\begin{array}{cc}
I_{n-1} \otimes L_{m-2}(1) & 0 \\
I_{n-1} \otimes R_{m-2}(1) & 0 \\
0 & L_{n-2}(1) \otimes I_{m-1} \\
0 & R_{n-2}(1) \otimes I_{m-1}
\end{array}\right) .
\end{aligned}
$$




\section{REFERENCES}

[1] L. Amodei And M. N. Benbourhim, A vector spline approximation, J. Approx. Theory, 67 (1991), pp. 51-79.

[2] G. Aubert, R. Deriche, and P. Kornprobst, Computing optical flow via variational techniques, SIAM J. Appl. Math., 60 (1999), pp. 156-182.

[3] J.-F. Aujol, G. Aubert, L. Blanc-Féraud, and A. Chambolle, Image decomposition application to SAR images, in Scale-Space Methods in Computer Vision, L. D. Griffin and M. Lilholm, eds. Lecture Notes in Comput. Sci. 2695, Springer, Berlin, 2003, pp. 297-312.

[4] J. F. Aujol and A. Chambolle, Dual norms and image decomposition models, Int. J. Computer Vision, 63 (2005), pp. 85-104.

[5] J. F. Aujol, G. Gilboa, T. Chan, and S. Osher Structure-texture image decompositionModeling, algorithms, and parameter selection, Internat. J. Computer Vision, 67 (2001), pp. 111-136.

[6] J. Bect, L. Blanc-Féraud, G. Aubert, and A. Chambolle, A $l_{1}$-unified variational framework for image restoration, in ECCV 2004, T. Pajdla and J. Matas, eds., Lecture Notes in Comput. Sci. 3024, Springer, Berlin-Heidelberg, 2004, pp. 1-13.

[7] A. Chambolle, An algorithm for total variation minimization and applications, J. Math. Imaging and Vision, 20 (2004), pp. 89-97.

[8] T. Chan, A. Marquina, and P. Mulet, High-order total variation-based image restoration, SIAM J. Sci. Comput., 22 (2000), pp. 503-516.

[9] T. Corpetti, E. MÉmin, And P. PÉRez, Dense estimation of fluid flows, IEEE Trans. Pattern Anal. and Machine Intell., 24 (2002), pp. 365-380.

[10] I. Daubechies And G. Teschke, Variational image restoration by means of wavelets: Simultaneous decomposition, deblurring and denoising, Appl. Comput. Harmonic Anal., 19 (2005), pp. 1-16.

[11] L. C. Evans and R. F. Gariepy, Measure Theory and Fine Properties of Functions, CRC Press, Boca Raton, FL, 1992.

[12] D. Goldfarb AND W. YIN, Second-order cone programming methods for total variation-based image restoration, SIAM J. Sci. Comput., 27 (2005), pp. 622-645.

[13] F. Guichard and L. Rudin, Accurate estimation of discontinuous optical flow by minimizing divergence related functionals, in Proceedings of the IEEE International Conference on Image Processing (ICIP), Lausanne, Switzerland, 1996, volume I, pp. 497-500.

[14] S. Gupta And J. Prince, Stochastic models for div-curl optical flow methods, Signal Process. Lett., 3 (1996), pp. 32-34.

[15] W. Hinterberger, O. Scherzer, C. Schnörr, and J. Weickert, Analysis of optical flow models in the framework of calculus of variations, Numer. Funct. Anal. Optim., 23 (2002), pp. 69-89.

[16] B. Horn and B. Schunck, Determining optical flow, Artificial Intell., 17 (1981), pp. 185-203.

[17] J. M. Hyman and M. J. Shashkov, Natural discretizations for the divergence, gradient, and curl on logically rectangular grids, Comput. Math. Appl., 33 (1997), pp. 81-104.

[18] M. C. Jullien, P. Castiglione, and P. Tabeling, Intermittency of a passive tracer in the inverse energy cascade, Phys. Rev. E, 64 (2001), paper 035301.

[19] J. Lewalle, J. Delville, and J.-P. Bonnet, Decomposition of mixed layer turbulence into coherent structures and background fluctations, Appl. Sci. Res., 64 (2000), pp. 301-328.

[20] S. B. M. S. Lobo, L. Vandenberghe, and H. Lebret, Applications of second order cone programming, Linear Algebra Appl., 284 (1998), pp. 193-228.

[21] Y. Meyer, Oscillating Patterns in Image Processing and Nonlinear Evolution Equations, University Lecture Series 22, AMS, Providence, RI, 2001.

[22] H. Mittelmann, An independent benchmarking of SDP and SOCP solvers, Math. Program. Ser. B, 95 (2003), pp. 407-430.

[23] M. Nikolova, Minimizers of cost-functions involving nonsmooth data-fidelity terms. Application to the processing of outliers, SIAM J. Numer. Anal., 40 (2002), pp. 965-994.

[24] S. Osher, A. Sole, AND L. A. VeSe, Image decomposition and restoration using total variation minimization and the $H^{-1}$ norm, Multiscale Model. Simul., 1 (2003), pp. 349-370.

[25] M. Raffel, C. Willert, and J. Kompenhans, Particle Image Velocimetry, 2nd ed., Springer, New York, 2001.

[26] L. I. Rudin, S. Osher, AND E. FATEmi, Nonlinear total variation based noise removal algorithms, Phys. D, 60 (1992), pp. 259-268.

[27] SeDuMi, Optimization software, http://sedumi.mcmaster.ca/.

[28] J.-L. Starck, M. Elad, AND D. L. Donoho, Image decomposition via the combination of sparse representations and a variational approach, IEEE Trans. Image Process., 14 (2005), pp. $1570-1582$. 
[29] G. STEId, A note on the dual treatment of higher order regularization functionals, Computing, 76 (2005), pp. 135-148.

[30] D. Suter, Motion estimation and vector splines, in Proceedings of the Conference on Computer Vision and Pattern Recognition, Los Alamos, CA, 1994, pp. 939-942.

[31] X.-C. TAi AND J. XU, Global and uniform convergence of subspace correction methods for some convex optimization problems, Math. Comput., 71 (2002), pp. 105-124.

[32] L. VeSE AND S. Osher, Modeling textures with total variation minimization and oscillating patterns in image processing, J. Sci. Comput., 19 (2003), pp. 553-572.

[33] J. Xu, Iterative methods by space decomposition and subspace correction, SIAM Rev., 34 (1992), pp. 581-613.

[34] W. Yin, D. Goldfarb, And S. Osher, Total Variation Based Image Cartoon-Texture Decomposition, Technical Report CORC TR-2005-01, Columbia University, New York, 2005.

[35] J. Yuan, R. Ruhnau, E. MÉmin, And C. SchnörR, Discrete orthogonal decomposition and variational fluid flow estimation, in Scale Space and PDE Methods in Computer Vision, Lecture Notes in Comput. Sci. 3459, Springer, New York, pp. 267-278.

[36] J. Yuan, C. SchnörR, G. Steidl, AND F. BeCKer, A study of non-smooth convex flow decomposition, in Proceedings of the Variational, Geometric and Level Set Methods in Computer Vision, Lecture Notes in Comput. Sci. 3752, Springer, New York, 2005, pp. $1-12$.

[37] J. YuAn, C. SChNÖRR, AND E. MÉmin, Discrete orthogonal decomposition and variational fluid flow estimation, J. Math. Imag. Vision, to appear. 